\title{
Balancedly splittable Hadamard matrices
}

\author{
Hadi Kharaghani* Sho Suda ${ }^{\dagger}$
}

October 18, 2018

\begin{abstract}
Balancedly splittable Hadamard matrices are introduced and studied. A connection is made to the Hadamard diagonalizable strongly regular graphs, maximal equiangular lines set, and unbiased Hadamard matrices. Several construction methods are presented. As an application, commutative association schemes of 4,5 , and 6 classes are constructed.
\end{abstract}

\section{Introduction}

An $n \times n$ matrix $H$ is a Hadamard matrix of order $n$ if its entries are $1,-1$ and it satisfies $H H^{\top}=I_{n}$, where $I_{n}$ denotes the identity matrix of order $n$. Hadamard matrices $H$ are shown to be related to other combinatorial objects such as combinatorial designs, distance regular graphs of diameter 4 , and the following under some regularity conditions:

- strongly regular graphs if $H$ is symmetric with constant diagonal [8],

- doubly regular tournaments if $H$ is skew-symmetric [22],

- symmetric or non-symmetric association schemes with 3 classes if $H$ is of symmetric or skew-symmetric Bush-type [9].

A Hadamard matrix $H$ of order $n$ is said to be balancedly splittable if there is an $\ell \times n$ submatrix $H_{1}$ of $H$ such that inner products for any two distinct column vectors of $H_{1}$ take at most two values. More precisely, there exist integers $a, b$ and the adjacency matrix $A$ of a graph such that $H_{1}^{\top} H_{1}=\ell I_{n}+a A+b\left(J_{n}-A-I_{n}\right)$, where $J_{n}$ denotes the all-ones matrix of order $n$.

We will show that the matrix $A$ is (switching equivalent)to the adjacency matrix of a strongly regular graph, and the graph is Hadamard diagonalizable in the sense of Barik, Fallat, and Kirkland [2]. The case $b=-a$ corresponds to a maximal equiangular lines set, and to the unbiased Hadamard matrices. We propose various constructions and provide non-existence results for balancedly splittable Hadamard matrices. A construction provided in Section 3.6 is related to recent work on complex Hadamard matrices [7] and the submatrix $H_{1}$ of a balancedly Hadamard matrix is essentially the same as the quasi-symmetric design constructed in [12]. It turns out that the property of balancedly splittable leads to a new relation between Hadamard matrices and various combinatorial objects.

As a further application we will make a connection to association schemes and provide schemes with 4, 5, 6-classes by using balancedly splittable Hadamard matrices and Latin squares. We will demonstrate that our approach relates to different concepts presented in [11, 13, 15, 16, [18, particularly in constructing unbiased Hadamard matrices and association schemes.

* Department of Mathematics and Computer Science, University of Lethbridge, Lethbridge, Alberta, T1K 3M4, Canada. kharaghani@uleth.ca

${ }^{\dagger}$ Department of Mathematics Education, Aichi University of Education, 1 Hirosawa, Igaya-cho, Kariya, Aichi, 448-8542, Japan. suda@auecc.aichi-edu.ac.jp 


\section{Balancedly splittable Hadamard matrices}

Definition 2.1. A Hadamard matrix $H$ of order $n$ is balancedly splittable if by suitably permuting its rows it can be transformed to $H=\left(\begin{array}{l}H_{1} \\ H_{2}\end{array}\right)$ such that the matrix $H_{1}^{\top} H_{1}$ has at most two distinct off-diagonal entries. In this case we say that $H$ is balancedly splittable with respect to $H_{1}$.

Let $H_{1}$ be an $\ell \times n$ matrix. Then there exist integers $a, b$ and a $(0,1)$-matrix $A$ such that $a \geq b$ and

$$
H_{1}^{\top} H_{1}=\ell I_{n}+a A+b\left(J_{n}-A-I_{n}\right) .
$$

The tuple of values $(n, \ell, a, b)$ is said to be the parameters of a balancedly splittable Hadamard matrix of order $n$ with respect to $H_{1}$.

By the equation $H_{1}^{\top} H_{1}+H_{2}^{\top} H_{2}=n I_{n}$, we have the following lemma.

Lemma 2.2. Let $H=\left(\begin{array}{l}H_{1} \\ H_{2}\end{array}\right)$ be a Hadamard matrix of order $n$ with $\ell \times n$ matrix $H_{1}, 1 \leq \ell<n$. Then $H$ is balancedly splittable with the parameters $(n, \ell, a, b)$ with respect to $H_{1}$ if and only if $H$ is balancedly splittable with the parameters $(n, n-\ell,-b,-a)$ with respect to $\mathrm{H}_{2}$.

The following are examples with $a=b$.

Example 2.3. (1) Any Hadamard matrix is balancedly splittable with respect to itself with the parameters $(n, n, 0,0)$.

(2) A Hadamard matrix $H=\left(\begin{array}{l}H_{1} \\ H_{2}\end{array}\right)$ of order $n$ with $H_{1}$ the all-ones vector is balancedly splittable with respect to $H_{1}$ with the parameters $(n, 1,1,1)$.

Conversely, it is easy to characterize a balancedly splittable Hadamard matrix to have $H_{1}^{\top} H_{1}$ with the only one distinct off-diagonal entry, as shown below.

Proposition 2.4. If a Hadamard matrix $H$ is balancedly splittable with $H_{1}^{\top} H_{1}=\ell I_{n}+a\left(J_{n}-I_{n}\right)$, then $(\ell, a) \in\{(1,1),(n-1,-1),(n, 0)\}$.

Proof. Squaring the equation $H_{1}^{\top} H_{1}=\ell I_{n}+a\left(J_{n}-I_{n}\right)$ yields that $n(\ell-a) I_{n}+n a J_{n}=(\ell-$ $a)^{2} I_{n}+\left(2(\ell-a) a+a^{2} n\right) J_{n}$. Comparing coefficients with $1 \leq \ell \leq n$, we have that $(\ell, a) \in$ $\{(1,1),(n-1,-1),(n, 0)\}$.

In the rest of the paper, we focus on balancedly splittable Hadamard matrices $H$ such that $H_{1}^{\top} H_{1}$ has exactly two distinct values off diagonal. The following is an obvious example.

Example 2.5. Let $H=\left(\begin{array}{l}H_{1} \\ H_{2}\end{array}\right)$ be a Hadamard matrix of order $n$ with $1 \times n$ matrix $H_{1}$. If $H_{1}$ is not equal to the all-ones vector, then $H$ is balancedly splittable with respect to $H_{1}$ with the parameters $(n, 1,1,-1)$.

Throughout the rest of the paper, we assume that $1<\ell<n-1$ in order to avoid the trivial cases.

A strongly regular graph with parameters $(v, k, \lambda, \mu)$ is a regular graph with $v$ vertices and degree $k$ such that every two adjacent (non-adjacent resp.) vertices have $\lambda$ ( $\mu$ resp.) common neighbors. The Seidel matrix of a graph with adjacency matrix $A$ is $S=J_{v}-I_{v}-2 A$. A strong 
graph is such that its Seidel matrix $S$ satisfies the property that $S^{2}$ is a linear combination of $S, I_{v}, J_{v}$. It is known that a graph is strongly regular if and only if it is regular and strong, see for [24] and [4, Chapter 10].

Balancedely splittable Hadamard matrices are related to strong graphs and strongly regular graphs, as shown in the following proposition.

Proposition 2.6. Let $H=\left(\begin{array}{l}H_{1} \\ H_{2}\end{array}\right)$ be a balancedly splittable Hadamard matrix of order $n$ with $H_{1}^{\top} H_{1}=\ell I_{n}+a A+b\left(J_{n}-A-I_{n}\right)$ where $A$ is an $n \times n(0,1)$-matrix, and $1<\ell<n-1, b<a$.

(1) If $b=-a, S=J_{n}-I_{n}-2 A$ is the Seidel matrix of a strong graph satisfying $S^{2}=$ $\frac{n-2 \ell}{a} S+\frac{\ell(n-\ell)}{a^{2}} I_{n}$ and $n=\frac{\ell^{2}-a^{2}}{\ell-a^{2}}$. The strong graph is switching equivalent to a strongly regular graph with the parameters $(n, k, \lambda, \mu)$ where $k, \lambda, \mu$ are the following:

$$
k=\frac{(a-1) \ell(a+\ell)}{2 a\left(\ell-a^{2}\right)}, \quad \lambda=\frac{(a+\ell)\left(3 a^{2}+a \ell-a-3 \ell\right)}{4 a\left(\ell-a^{2}\right)}, \quad \mu=\frac{(a-1)\left(\ell^{2}-a^{2}\right)}{4 a\left(\ell-a^{2}\right)} .
$$

(2) If $b \neq-a$, then $A$ is the adjacency matrix of a strongly regular graph with parameters $(n, k, \lambda, \mu)$, where $b, k, \lambda, \mu$ are either one of the following:

(a) $b=\frac{\ell(-a+\ell-n)}{a(n-1)+\ell}, \quad k=\frac{\ell n(n-\ell-1)}{n\left(a^{2}+\ell\right)-(a-\ell)^{2}}, \lambda=\frac{n\left(n^{2}\left(a^{3}+\ell^{2}\right)-2(\ell+1) n\left(a^{3}+\ell^{2}\right)+(2 a \ell+a+\ell(\ell+2))(a-\ell)^{2}\right)}{\left((a-\ell)^{2}-n\left(a^{2}+\ell\right)\right)^{2}}$, $\mu=\frac{\ln (a-\ell)(\ell-n+1)(a-\ell+n)}{\left((a-\ell)^{2}-n\left(a^{2}+\ell\right)\right)^{2}}$.

(b) $b=\frac{(a-\ell)(\ell-n)}{a(n-1)+\ell-n}, k=\frac{(\ell-1) n(\ell-n)}{(a-\ell)^{2}-n((a-2) a+\ell)}, \lambda=\frac{n\left(a^{3}\left(-2 \ell(n-1)+n^{2}-1\right)-3 a^{2}(\ell-n)^{2}+3 a(\ell-n)^{2}+(\ell-2) \ell(\ell-n)^{2}\right)}{\left((a-\ell)^{2}-n((a-2) a+\ell)\right)^{2}}$, $\mu=\frac{(\ell-1) n(a-\ell)(\ell-n)(a-\ell+n)}{\left((a-\ell)^{2}-n((a-2) a+\ell)\right)^{2}}$.

Furthermore if (a) occurs, then each row of $H_{1}$ is orthogonal to the all-ones vector.

Proof. Squaring (2.1) with the fact that $H_{1} H_{1}^{\top}=n I_{\ell}$, we have that

$$
\left(\ell I_{n}+a A+b\left(J_{n}-A-I_{n}\right)\right)^{2}=n\left(\ell I_{n}+a A+b\left(J_{n}-A-I_{n}\right)\right) .
$$

Simplifying (2.3) by $b \neq a$ yields that

$$
\begin{aligned}
A^{2}=\frac{1}{(a-b)^{2}}( & (a-b)(n-2 \ell+2 b) A \\
& \left.+(\ell-b)(n-\ell+b) I_{n}+b(n-n b-2 \ell+2 b) J_{n}-(a-b) b\left(A J_{n}+J_{n} A\right)\right) .
\end{aligned}
$$

For $x \in\{1, \ldots, n\}$, let $k_{x}$ denote the degree of $x$ in the graph whose adjacency matrix is $A$. Then comparing the $(x, x)$-entry in (2.3) shows that

$$
\ell^{2}+a^{2} k_{x}+b^{2}\left(n-1-k_{x}\right)=n \ell .
$$

(1): For the case $b=-a$, by $H_{1}^{\top} H_{1}=\ell I_{n}+a S$, (2.3) is reduced to $S^{2}=\frac{n-2 \ell}{a} S+\frac{\ell(n-\ell)}{a^{2}} I_{n}$, and (2.5) shows that $\ell^{2}+a^{2}(n-1)=n \ell$. Since $\ell \neq 1$, we have that $\ell \neq a^{2}$. Thus $n=\frac{\ell^{2}-a^{2}}{\ell-a^{2}}$.

Normalize the Hadamard matrix $H$ so that the last row of $H$ equals to the all-ones vector. Then multiplying $J_{n}$ by $H_{1}^{\top} H_{1}=(\ell+a) I_{n}+2 a A-a J_{n}$, we have $2 a A J_{n}=(a n-\ell-a) J_{n}$. Since $a \neq 0$, the graph is regular with valency $k=\frac{a n-\ell-a}{2 a}$. The strong graph with the Seidel matrix $S$ is regular, and thus it is strongly regular. Let $(n, k, \lambda, \mu)$ be its parameters. The parameters are determined as in (2.2) by substituting $b=-a$ and $A J_{n}=J_{n} A=\frac{a n-\ell-a}{2 a} J_{n}$ into (2.4) with use of $n=\frac{\ell^{2}-a^{2}}{\ell-a^{2}}$. 
(2): By the assumption that $b \neq \pm a$, (2.5) shows that $k_{x}=\frac{n \ell-\ell^{2}-b^{2}(n-1)}{a^{2}-b^{2}}$, which is independent of the particular choice of $x$. Thus $A$ is the adjacency matrix of a regular graph of degree $k$ given as

$$
k:=\frac{n \ell-\ell^{2}-b^{2}(n-1)}{a^{2}-b^{2}} .
$$

To use the fact that $A J_{n}=J_{n} A=k J_{n}$, (2.4) shows that the matrix $A$ is the adjacency matrix of a strongly regular graph with parameters $(n, k, \lambda, \mu)$ where $\lambda, \mu$ are determined as follows:

$$
\begin{aligned}
& \lambda=\frac{n\left(a^{2}-a(b-1) b+b^{3}-2 b \ell\right)+2(b-\ell)\left(a^{2}+a b-b(b+\ell)\right)}{(a-b)^{2}(a+b)}, \\
& \mu=\frac{b n\left(-a b+a+b^{2}+b-2 \ell\right)+2 b(a-\ell)(b-\ell)}{(a-b)^{2}(a+b)} .
\end{aligned}
$$

Substituting (2.7), (2.8) into the well-known formula $k(k-\lambda-1)=(n-k-1) \mu$ and simplifying it, we have

$$
((a(n-1)+\ell) b-(-a+\ell-n))((a(n-1)+\ell-n) b-(a-\ell)(\ell-n))=0 .
$$

Since $a(n-1)+\ell>0$ and $a(n-1)+\ell-n>0$, (2.9) implies that $b=\frac{\ell(-a+\ell-n)}{a(n-1)+\ell}$ or $b=\frac{(a-\ell)(\ell-n)}{a(n-1)+\ell-n}$. Thus by (2.6), (2.7), (2.8) we obtain the desired formula for $k, \lambda$ and $\mu$.

For (a), pre-multiplying the all-ones column vector 1 and post-multiplying its transpose by (2.1) shows that

$$
\left(H_{1} \mathbf{1}\right)^{\top}\left(H_{1} \mathbf{1}\right)=\mathbf{1}^{\top}\left(\ell I_{n}+a A+b\left(J_{n}-A-I_{n}\right)\right) \mathbf{1}=(\ell+a k+b(n-1-k)) n=0,
$$

where we used $b=\frac{\ell(-a+\ell-n)}{a(n-1)+\ell}, k=\frac{\ell n(n-\ell-1)}{n\left(a^{2}+\ell\right)-(a-\ell)^{2}}$ in the last equality. Thus $H_{1} \mathbf{1}=\mathbf{0}$ holds where $\mathbf{0}$ is the zero vector.

Remark 2.7. It is routinely checked that the parameters of the strongly regular graphs in (2) (a) are valid for the case (1) to use $\frac{\ell(n-\ell)}{a^{2}}=n-1$.

Remark 2.8. Let $H=\left(\begin{array}{l}H_{1} \\ H_{2}\end{array}\right)$ be a Hadamard matrix of order $n$.

(1) It is easy to check that $H$ is balancedly splittable with the parameters $(n, \ell, a, b)$ with respect to $H_{1}$ fitting into Proposition 2.6(2)(a) if and only if $H$ is balancedly splittable with the parameters $(n, n-\ell,-b,-a)$ with respect to $H_{2}$ fitting into Proposition 2.6 (2)(b).

(2) Assume that $H$ is a balancedly splittable Hadamard matrix of order $n$ with the parameters $(n, \ell, a,-a)$ with respect to $H_{1}$ and the last row being the all-ones vector. Then the Hadamard matrix $H$ is a balancedly splittable Hadamard matrix of order $n$ with the parameters $(n, n-\ell-1, a-1,-a-1)$ with respect to the submatrix of $H$ obtained by deleting $H_{1}$ and the last row from $H$.

Remark 2.9. A strongly regular graph is said to be imprimitive if either the graph or its complement is disconnected. This is equivalent to $k=\lambda+1$ or $k=\mu$. The former occurs in (2)(a) if and only if $a=\ell$. The latter occurs in (2)(a) if and only if $a=0$.

A graph is said to be Hadamard diagonalizable if its Laplacian matrix $L$ is diagonalized by a Hadamard matrix, that is, there exists a Hadamard matrix $H$ such that $H L H^{\top}$ is a diagonal matrix [2]. It turns out that a Hadamard diagonalizable graph is regular [2, Theorem 5]. Therefore a graph is Hadamard diagonalizable if and only if its adjacency matrix is diagonalized by a Hadamard matrix. 
Corollary 2.10. (1) If a Hadamard matrix $H=\left(\begin{array}{l}H_{1} \\ H_{2}\end{array}\right)$ of order $n$ is balancedly splittable with respect to $H_{1}$ with parameters $(n, \ell, a, b)$ such that $H_{2}$ has the all-ones row vector, then the strongly regular graph constructed in Theorem 2.6 is Hadamard diagonalizable by $H$.

(2) Conversely, if a strongly regular graph on $n$ vertices which is Hadamard diagonalizable by a normalized Hadamard matrix $H$, then $H$ is balancedly splittable with parameters $(n, \ell, a, b)$ where

Proof. Assume (1) to be true. It holds that

$$
H H_{1}^{\top} H_{1} H^{\top}=\operatorname{diag}(\underbrace{n^{2}, \ldots, n^{2}}_{\ell}, \underbrace{0, \ldots, 0}_{n-\ell}) .
$$

Without loss of generality, we may assume that the last row of $H_{2}$ is the all-ones vector. Then we have that $H J_{n} H^{\top}=\operatorname{diag}\left(0, \ldots, 0, n^{2}\right)$. Pre-multiplying $H$ and post-multiplying $H^{\top}$ by (2.1) and simplifying it yields that

$$
H A H^{\top}=\frac{n}{a-b} \operatorname{diag}(\underbrace{-\ell+b+n, \ldots,-\ell+b+n}_{\ell}, \underbrace{-\ell+b, \ldots,-\ell+b}_{n-\ell-1},-\ell-b(n-1)) .
$$

Therefore $A$ is Hadamard diagonalizable by $H$.

Conversely assume (2), namely let $A$ be a strongly regular graph which is diagonalized by a normalized Hadamard matrix $H$ of order $n$. Without loss of generality $H$ has the all-ones vector as the last row. Then it holds that by a suitable rearranging rows and columns of $A$,

$$
H A H^{\top}=\operatorname{diag}(\underbrace{\theta, \ldots, \theta}_{\ell}, \underbrace{\tau, \ldots, \tau}_{n-\ell-1}, k)
$$

where $k$ is the valency of $A$, and $\theta, \tau$ are distinct eigenvalues of $A$ (one of which may be equal to $k$ ), and $\ell$ is the multiplicity of $\theta$. Write $H=\left(\begin{array}{l}H_{1} \\ H_{2}\end{array}\right)=\left(\begin{array}{l}H_{1} \\ H_{2}^{\prime} \\ \mathbf{1}^{\top}\end{array}\right)$ where $H_{1}$ is the $\ell \times n$ matrix and $H_{2}$ is the $(n-\ell) \times n$ matrix and $H_{2}^{\prime}$ is the $(n-\ell-1) \times n$ submatrix of $H_{2}$. Pre-multiplying $H^{\top}$ and post-multiplying $H$ by (2.10) provides

$$
n^{2} A=\theta H_{1}^{\top} H_{1}+\tau\left(H_{2}^{\prime}\right)^{\top} H_{2}^{\prime}+k J_{n} .
$$

On the other hand, by $H^{\top} H=n I$, we have

$$
n I_{n}=H_{1}^{\top} H_{1}+\left(H_{2}^{\prime}\right)^{\top} H_{2}^{\prime}+J_{n} .
$$

Therefore by (2.11) and (2.12) we have that

$$
H_{1}^{\top} H_{1}=\frac{1}{\theta-\tau}\left(n^{2} A-\tau n I_{n}-(k-\tau) J_{n}\right) .
$$

Thus a Hadamard matrix $H=\left(\begin{array}{l}H_{1} \\ H_{2}\end{array}\right)$ is balancedly splittable with respect to the $\ell \times n$ matrix $H_{1}$ such that $H_{2}$ has the all-ones row vector.

We list the feasible parameters in Table 1 for (1) with $n \leq 1024, \ell \leq n / 2$ and those in Table 2 for (2)(a) with $n \leq 64$ and $0<a<\ell$. In the tables, E stands for "exists" and NE stands for "does not exist".

The following upper bound is due to Delsarte, Goethals and Seidel [5]. The finite set $X$ of $\mathbb{R}^{m}$ satisfying the assumption in Proposition 2.11 is referred to as an equiangular lines set. 
Table 1: $n \leq 1024, \ell \leq n / 2$

\begin{tabular}{cccc|cccc}
$n$ & $\ell$ & $a$ & & $n$ & $\ell$ & $a$ & \\
\hline 16 & 6 & 2 & E & 528 & 187 & 11 & NE, Prop 2.16 \\
36 & 15 & 3 & NE, Prop 2.16 & 540 & 99 & 9 & NE, Prop 2.17 \\
64 & 28 & 4 & E & 560 & 130 & 10 & \\
100 & 45 & 5 & NE, Prop 2.16 & 576 & 276 & 12 & \\
120 & 35 & 5 & NE, Prop 2.17 & 616 & 165 & 11 & NE, Prop 2.17 \\
144 & 66 & 6 & & 640 & 72 & 8 & \\
196 & 91 & 7 & NE, Prop 2.16 & 676 & 325 & 13 & NE, Prop 2.16 \\
256 & 120 & 8 & E & 780 & 247 & 13 & NE, Prop 2.17 \\
280 & 63 & 7 & NE, Prop 2.16 & 784 & 378 & 14 & \\
288 & 42 & 6 & & 900 & 435 & 15 & NE, Prop 2.16 \\
320 & 88 & 8 & 924 & 143 & 11 & NE, Prop 2.16 \\
324 & 153 & 9 & NE, Prop 2.16 & 936 & 221 & 13 & NE, Prop 2.16 \\
400 & 190 & 10 & & 1008 & 266 & 14 & \\
484 & 231 & 11 & NE, Prop 2.16 & 1024 & 496 & 16 & E
\end{tabular}

Table 2: $n \leq 64$ and $0<a<\ell$

\begin{tabular}{cccccccc}
$n$ & $\ell$ & $a$ & $b$ & $k$ & $\lambda$ & $\mu$ & \\
\hline 16 & 5 & 1 & -3 & 10 & 6 & 6 & E, Remark 2.8(2) \\
16 & 9 & 1 & -3 & 9 & 4 & 6 & E, Remark 2.8(2) \\
36 & 10 & 4 & -2 & 10 & 4 & 2 & NE, Prop 2.21 (1) \\
36 & 14 & 2 & -4 & 21 & 12 & 12 & NE, Prop 2.21] (3) \\
36 & 20 & 2 & -4 & 20 & 10 & 12 & NE, Prop 2.21] $(4)$ \\
36 & 25 & 1 & -5 & 25 & 16 & 20 & NE, Prop 2.21 $(2)$ \\
64 & 14 & 6 & -2 & 14 & 6 & 2 & E, Theorem 3.1 \\
64 & 18 & 2 & -6 & 45 & 32 & 30 & \\
64 & 21 & 5 & -3 & 21 & 8 & 6 & \\
64 & 27 & 3 & -5 & 36 & 20 & 20 & E, Remark 2.8 (2) \\
64 & 35 & 3 & -5 & 35 & 18 & 20 & E, Remark 2.8(2) \\
64 & 42 & 2 & -6 & 42 & 26 & 30 & \\
64 & 45 & 5 & -3 & 18 & 2 & 6 & \\
64 & 49 & 1 & -7 & 49 & 36 & 42 & E, Theorem 3.1
\end{tabular}


Proposition 2.11. Let $X \subset \mathbb{R}^{m}$ be a set of unit vectors such that $|\langle v, w\rangle|=\alpha$ for all $v, w \in$ $X, v \neq w$. If $m<\frac{1}{\alpha^{2}}$, then

$$
|X| \leq \frac{m\left(1-\alpha^{2}\right)}{1-m \alpha^{2}}
$$

Proposition 2.12. If there exists a balancedly splittable Hadamard matrix with the parameters $(n, \ell, a,-a)$, then there exists an equiangular lines set in $\mathbb{R}^{\ell}$ with inner product $\frac{\sqrt{n-\ell}}{\sqrt{\ell(n-1)}}$ attaining equality in (2.13).

Proof. Let $H=\left(\begin{array}{l}H_{1} \\ H_{2}\end{array}\right)$ be a balancedly Hadamard matrix with respect to an $\ell \times n$ matrix $H_{1}$. Let $X$ be the set of column vectors of $H_{1}$ normalized by dividing by $\sqrt{\ell}$, so $a^{2} \ell^{2}=\frac{n \ell-\ell^{2}}{n-1}$. Then $X$ is a subset of $n$ unit vectors of $\mathbb{R}^{\ell}$ such that $|\langle v, w\rangle|=\frac{\sqrt{n-\ell}}{\sqrt{\ell(n-1)}}$ for all $v, w \in X, v \neq w$. It can be seen that $m<\frac{1}{\alpha^{2}}$ for $(m, \alpha)=\left(\ell, \frac{\sqrt{n-\ell}}{\sqrt{\ell(n-1)}}\right)$ and that the right hand side in (2.13) in this case equals to $n$. Thus our equiangular lines set attains the bound in (2.13).

Two Hadamard matrices $H$ and $K$ of order $n$ are said to be unbiased if $\frac{1}{\sqrt{n}} H K^{\top}$ is a Hadamard matrix of order $n$.

Proposition 2.13. Let $H=\left(\begin{array}{l}H_{1} \\ H_{2}\end{array}\right)$ be a balancedly splittable Hadamard matrix of order $n$ with $H_{1}^{\top} H_{1}=\ell I_{n}+a S$ where $a \neq 0$ and $S$ is an $n \times n(0,1,-1)$-matrix. Then the following are equivalent.

(1) $K:=\frac{1}{2 a}\left(H_{1}^{\top} H_{1}-H_{2}^{\top} H_{2}\right)$ is a Hadamard matrix.

(2) $(\ell, a)=((n \pm \sqrt{n}) / 2, \sqrt{n} / 2)$.

In this case, $n=4 k^{2}$ for some integer $k$ and the Hadamard matrices $H$ and $K$ are unbiased.

Proof. Since $H_{1} H_{2}^{\top}$ and $H_{2}^{\top} H_{1}^{\top}$ are zero matrices and $H_{1} H_{1}^{\top}=n I_{\ell}, H_{2} H_{2}^{\top}=n I_{n-\ell}$,

$$
\begin{aligned}
K K^{\top} & =K^{2}=\frac{1}{4 a^{2}}\left(H_{1}^{\top} H_{1}-H_{2}^{\top} H_{2}\right)^{2}=\frac{1}{4 a^{2}}\left(H_{1}^{\top}\left(H_{1} H_{1}^{\top}\right) H_{1}+H_{2}^{\top}\left(H_{2} H_{2}^{\top}\right) H_{2}\right) \\
& =\frac{n}{4 a^{2}}\left(H_{1}^{\top} H_{1}+H_{2}^{\top} H_{2}\right)=\frac{n^{2}}{4 a^{2}} I_{n} .
\end{aligned}
$$

Therefore $K$ is a Hadamard matrix if and only if $K$ is a $(1,-1)$-matrix and $a=\sqrt{n} / 2$. Since $K=$ $\frac{1}{2 a}\left(H_{1}^{\top} H_{1}-H_{2}^{\top} H_{2}\right)=\frac{1}{2 a}\left((2 \ell-n) I_{n}-2 a S\right), K$ is a $(1,-1)$-matrix if and only if $(2 \ell-n) /(2 a)= \pm 1$. By Proposition 2.6(1) the latter is equivalent to $\ell=(n \pm \sqrt{n}) / 2$. Therefore (1) is equivalent to (2).

If (1) and (2) hold, then $a=\sqrt{n} / 2$ is an integer. Therefore $n$ must be a square of an even integer. And we have that $H K^{\top}=\sqrt{n}\left(\begin{array}{c}H_{1} \\ -H_{2}\end{array}\right)$. Thus $H$ and $K$ are unbiased.

A Hadamard matrix of order $n$ is said to be regular if $\mathbf{1}^{\top} H=\sqrt{n} \mathbf{1}^{\top}$. In this case $n$ must be square.

Proposition 2.14. Any balanced splittable Hadamard matrix of order $4 n^{2}$ with the parameters $(\ell, a, b)=\left(2 n^{2}-n, n,-n\right)$ is equivalent to a regular Hadamard matrix. 
Proof. Let $H=\left(\begin{array}{l}H_{1} \\ H_{2}\end{array}\right)$ where $H_{1}$ is an $\ell \times n$ matrix. Since multiplying a signed permutation matrix by $H$ from the left keeps the property of balancedly splittable, we may assume that $H_{1}$ has the all-ones first column and $H_{2}$ has the negative all-ones first column. By the assumption $b=-a$, multiplying a signed permutation matrix by $H$ from the right also keeps the property of balancedly splittable. This implies that $\mathbf{1}^{\top} H_{1}=\left(-2 n^{2}+n, n, \ldots, n\right)$ and $\mathbf{1}^{\top} H_{2}=\left(2 n^{2}+n, n, \ldots, n\right)$. Therefore $\mathbf{1}^{\top} H=\mathbf{1}^{\top} H_{1}+\mathbf{1}^{\top} H_{2}=(2 n, 2 n, \ldots, 2 n)$, which proves that $H$ is equivalent to a regular Hadamard matrix.

Remark 2.15. The Hadamard matrices of order 16 with Hall's classes IV or V are not equivalent to regular Hadamard matrices, and thus are not balancedly splittable with the parameters $(\ell, a,-b)=(6,2,-2)$ [10]. See [26] for Hall's classes of Hadamard matrices.

We now present two non-existence results.

Proposition 2.16. There is no balancedly splittable Hadamard matrix with the parameters $(n, \ell, a,-a), \ell+a \not \equiv 0(\bmod 4), 1<\ell<n-1$.

Proof. Assume that there exists such a balancedly Hadamard matrix $H=\left(\begin{array}{l}H_{1} \\ H_{2}\end{array}\right)$ where $H_{1}$ is an $\ell \times n$ matrix. By multiplying $H$ on both sides by signed permutation matrices, we may assume that $H_{1}^{\top} H_{1}$ has $a$ as its entries in the first row and the first column except $(1,1)$-entry. Now we claim that $H_{1}^{\top} H_{1}=\ell I+a(J-I)$. Indeed, suppose for the contrary that there exist two columns, say $i$-th and $j$-th columns, distinct from the first column such that their inner product equals to $-a$. Let $x, y, x, w$ be non-negative integers such that

$$
\begin{aligned}
& \text { the first column }=(+\cdots++\cdots++\cdots++\cdots+)^{\top}, \\
& \text { the } i \text {-th column }=(+\cdots++\cdots+-\cdots--\cdots-)^{\top}, \\
& \text { the } j \text {-th column }=(\underbrace{+\cdots+}_{x \text { rows }} \underbrace{-\cdots-}_{y \text { rows }} \underbrace{+\cdots+}_{z \text { rows }} \underbrace{-\cdots-}_{w \text { rows }})^{\top} .
\end{aligned}
$$

Then it follows that

$$
\left\{\begin{array}{l}
x+y+z+w=\ell, \\
x+y-z-w=a, \\
x-y+z-w=a, \\
x-y-z+w=-a .
\end{array}\right.
$$

Solving these equations yields $(x, y, z, w)=\left(\frac{\ell+a}{4}, \frac{\ell+a}{4}, \frac{\ell+a}{4}, \frac{\ell-3 a}{4}\right)$, which is impossible because $\ell+a \not \equiv 0(\bmod 4)$. Therefore we have $H_{1}^{\top} H_{1}=\ell I+a(J-I)$.

However, $H_{1}^{\top} H_{1}=\ell I+a(J-I)$ contradicts Proposition 2.4 by $1<\ell<n-1$. Therefore we conclude that such a balancedly splittable Hadamard matrix does not exist.

In a similar way the following is proved.

Proposition 2.17. There is no balancedly splittable Hadamard matrix with the parameters $(n, \ell, a,-a), \ell \neq \equiv a(\bmod 4)$ and $a>1$.

Proof. In the same way as in Proposition 2.16 we may assume that there exists such a balancedly Hadamard matrix $H=\left(\begin{array}{l}H_{1} \\ H_{2}\end{array}\right)$ where $H_{1}$ is an $\ell \times n$ matrix and $H_{1}^{\top} H_{1}$ has $a$ as its entries in the first row and the first column except $(1,1)$-entry. Now we claim that

$$
H_{1}^{\top} H_{1}=\left(\begin{array}{cc}
\ell & a \mathbf{1}^{\top} \\
a \mathbf{1} & \ell I_{n-1}-a\left(J_{n-1}-I_{n-1}\right)
\end{array}\right) .
$$


Indeed, suppose to the contrary that there exist two columns, say $i$-th and $j$-th columns, distinct from the first column such that their inner product equals to $a$. Let $x, y, x, w$ be non-negative integers such that

$$
\begin{aligned}
& \text { the first column }=(+\cdots++\cdots++\cdots++\cdots+)^{\top} \text {, } \\
& \text { the } i \text {-th column }=(+\cdots++\cdots+-\cdots--\cdots-)^{\top} \text {, } \\
& \text { the } j \text {-th column }=(\underbrace{+\cdots+}_{x \text { rows }} \underbrace{-\cdots-}_{y \text { rows }} \underbrace{+\cdots+}_{z \text { rows }} \underbrace{-\cdots-}_{w \text { rows }})^{\top} \text {. }
\end{aligned}
$$

Then It is seen that

$$
\left\{\begin{array}{l}
x+y+z+w=\ell \\
x+y-z-w=a \\
x-y+z-w=a \\
x-y-z+w=a .
\end{array}\right.
$$

Solving these equations yields $(x, y, z, w)=\left(\frac{\ell+3 a}{4}, \frac{\ell-a}{4}, \frac{\ell-a}{4}, \frac{\ell-a}{4}\right)$, which is impossible because $l \not \equiv a(\bmod 4)$. Therefore we have $H_{1}^{\top} H_{1}=\left(\begin{array}{cc}\ell & a \mathbf{1}^{\top} \\ a \mathbf{1} & \ell I_{n-1}-a\left(J_{n-1}-I_{n-1}\right)\end{array}\right)$. It can beseen that $\ell-(n-1) a$ is one of the eigenvalues of $\left(\begin{array}{cc}\ell & a \mathbf{1}^{\top} \\ a \mathbf{1} & \ell I_{n-1}-a\left(J_{n-1}-I_{n-1}\right)\end{array}\right)$ However, $\ell-(n-1) a<0$ for $a>1$, which contradicts the fact that all the singular values of $H_{1}$ are nonnegative. Therefore we conclude that such a balancedly splittable Hadamard matrix does not exist.

There are exactly three inequivalent Hadamard matrices of order 16 with maximal excess 64 . Those are contained in the Hall's classes I, II, and III. The corresponding strongly regular graphs are $K_{4} \times K_{4}$ and the Shrikhande graph [23]). These three Hadamard matrices are balancedly splittable. The following are three examples of order 16.

Example 2.18. The Hadamard matrix of order 16 of Hall's class I, that is, the Sylvester Hadamard matrix is balancedely splittable with parameters $(16,6,2,-2)$. The corresponding strongly regular graph is $K_{4} \times K_{4}$.

Example 2.19. The Hadamard matrix of order 16 of Hall's class II is balancedely splittable with parameters $(16,6,2,-2)$. The corresponding strongly regular graph is $K_{4} \times K_{4}$.

Example 2.20. The Hadamard matrix of order 16 of Hall's class III is balancedely splittable with parameters $(16,6,2,-2)$. The corresponding strongly regular graph is the Shrikhande graph [23].

Though the classification of Hadamard matrices of order 36 has not been finished yet, we have the non-existence results for balancedly splittable Hadamard matrices of order 36 by dealing with the eigenspaces of the attached strongly regular graphs.

Proposition 2.21. (1) There is no balancedely splittable Hadamard matrix of order 36 with the parameters $(36,10,4,-2)$.

(2) There is no balancedely splittable Hadamard matrix of order 36 with the parameters $(36,25,1,-5)$.

(3) There is no balancedely splittable Hadamard matrix of order 36 with the parameters $(36,14,2,-4)$.

(4) There is no balancedely splittable Hadamard matrix of order 36 with the parameters $(36,20,2,-4)$. 
Proof. (1): If there would exist such a Hadamard matrix $H$, then $H$ must come from the unique strongly regular graph with the parameters $(36,10,4,2)$ having the adjacency matrix $A$. The matrix $B:=12 I+6 A-2 J$ is written as $12 I+6 A-2 J=H_{1}^{\top} H_{1}$ where $H_{1}$ is a $10 \times 36(1,-1)$ matrix. Then the eigenvectors of $B$ with eigenvalue 36 are the row vectors of the matrix $\left(I_{10}, X\right)$ where $X$ is

$$
\left(\begin{array}{cccccccccccccccccccccccccc}
4 & - & - & - & - & 3 & - & - & - & - & 3 & - & - & - & - & 3 & - & - & - & - & 3 & - & - & - & - & 3 \\
- & 1 & 1 & 1 & 1 & 0 & 0 & 0 & 0 & 0 & - & 0 & 0 & 0 & 0 & - & 0 & 0 & 0 & 0 & - & 0 & 0 & 0 & 0 & - \\
- & 0 & 0 & 0 & 0 & - & 1 & 1 & 1 & 1 & 0 & 0 & 0 & 0 & 0 & - & 0 & 0 & 0 & 0 & - & 0 & 0 & 0 & 0 & - \\
- & 0 & 0 & 0 & 0 & - & 0 & 0 & 0 & 0 & - & 1 & 1 & 1 & 1 & 0 & 0 & 0 & 0 & 0 & - & 0 & 0 & 0 & 0 & - \\
-0 & 0 & 0 & 0 & - & 0 & 0 & 0 & 0 & - & 0 & 0 & 0 & 0 & - & 1 & 1 & 1 & 1 & 0 & 0 & 0 & 0 & 0 & - \\
- & 0 & 0 & 0 & 0 & - & 0 & 0 & 0 & 0 & - & 0 & 0 & 0 & 0 & - & 0 & 0 & 0 & 0 & - & 1 & 1 & 1 & 1 & 0 \\
-1 & 0 & 0 & 0 & - & 1 & 0 & 0 & 0 & - & 1 & 0 & 0 & 0 & - & 1 & 0 & 0 & 0 & - & 1 & 0 & 0 & 0 & - \\
- & 0 & 1 & 0 & 0 & - & 0 & 1 & 0 & 0 & - & 0 & 1 & 0 & 0 & - & 0 & 1 & 0 & 0 & - & 0 & 1 & 0 & 0 & - \\
- & 0 & 0 & 1 & 0 & - & 0 & 0 & 1 & 0 & - & 0 & 0 & 1 & 0 & - & 0 & 0 & 1 & 0 & - & 0 & 0 & 1 & 0 & - \\
- & 0 & 0 & 0 & 1 & - & 0 & 0 & 0 & 1 & - & 0 & 0 & 0 & 1 & - & 0 & 0 & 0 & 1 & - & 0 & 0 & 0 & 1 & -
\end{array}\right),
$$

and - stands for -1 . By computer search, there are no mutually orthogonal 10 eigenvectors of $B$ with eigenvalue 36 and entries $1,-1$. Therefore there is no Hadamard matrix $H$ of order 36 such that any $10 \times 36$ submatrix $H_{1}$ of $H$ satisfies that $12 I+6 A-2 J=H_{1}^{\top} H_{1}$.

The proofs for (2), (3), and (4) are the same as that of (1).

Note that the strongly regular graph for (2) is the complement of that for (1). There exist 180 strongly regular graphs with the parameters $(36,21,12,12)$ which correspond to the case (3), and there exist 32548 strongly regular graphs with the parameters $(36,20,10,12)$ which correspond to the case (4).

\section{Constructions}

In this section, we construct several balancedly splittable Hadamard matrices.

3.1 Construction for $(n, \ell, a, b)=\left(m^{2},(m-1)^{2}, 1,-m+1\right),\left(m^{2}, 2 m-2, m-2,-2\right)$, $m$ an order for a Hadamard matrix

Theorem 3.1. Let $m$ be the order for a Hadamard matrix. Then there exists a balancedly splittable Hadamard matrix of order $m^{2}$ with the parameters $\left(m^{2},(m-1)^{2}, 1,-m+1\right)$ and $\left(m^{2}, 2 m-2, m-2,-2\right)$.

Proof. Let $H$ be a Hadamard matrix of order $m$. Normalize $H$ so that $H=\left(\begin{array}{l}\mathbf{1}^{\top} \\ H_{1}\end{array}\right)$. Then $H \otimes H$ is a Hadamard matrix and has $H_{1} \otimes H_{1}$ as a submatrix of $H \otimes H$. Then using the property that $H_{1}^{\top} H_{1}=m I_{m}-J_{m}$, we have

$$
\left(H_{1} \otimes H_{1}\right)^{\top}\left(H_{1} \otimes H_{1}\right)=H_{1}^{\top} H_{1} \otimes H_{1}^{\top} H_{1}=\left(m I_{m}-J_{m}\right) \otimes\left(m I_{m}-J_{m}\right),
$$

which has only two distinct entries off diagonal. Therefore $H \otimes H$ is a balancedly splittable Hadamard matrix of order $m^{2}$ with the parameters $\left(m^{2},(m-1)^{2}, 1,-m+1\right)$. Note that $H \otimes H$ is normalized and the all-ones row vector is not a row vector of $H_{1} \otimes H_{1}$. Then we use the fact in Remark 2.8 (2) to show that $H \otimes H$ is also a balancedly splittable Hadamard matrix of order $m^{2}$ with the parameters $\left(m^{2}, 2 m-2, m-2,-2\right)$.

3.2 Construction for $(n, \ell, a, b)=\left(m^{2}, m, m, 0\right), m$ an order for a Hadamard matrix

Theorem 3.2. Let $m$ be the order for a Hadamard matrix. Then there exists a balancedly splittable Hadamard matrix of order $m^{2}$ with the parameters $\left(m^{2}, m, m, 0\right)$. 
Proof. Let $H$ be a Hadamard matrix of order $m$. Let $r_{i}$ be the $i$-th row of $H$ for $i \in\{1, \ldots, m\}$ and normalize $H$ so that $r_{1}$ is the all-ones vector. Define an $m^{2} \times m^{2}$ matrix $M$ by $M=$ $\left(r_{j}^{\top} r_{i}\right)_{i, j=1}^{m}$. Then $M$ is a Hadamard matrix of order $m^{2}$. Let $M_{1}=\left(r_{j}^{\top} r_{1}\right)_{j=1}^{m}$ be a submatrix of M. By $r_{i} r_{j}^{\top}=m \delta_{i, j}$ and $r_{1}=\mathbf{1}_{m}^{\top}$, we have

$$
M_{1}^{\top} M_{1}=\left(\begin{array}{c}
r_{1}^{\top} r_{1} \\
r_{1}^{\top} r_{2} \\
\vdots \\
r_{1}^{\top} r_{m}
\end{array}\right)\left(r_{1}^{\top} r_{1}, r_{2}^{\top} r_{1}, \ldots, r_{m}^{\top} r_{1}\right)=\left(\begin{array}{cccc}
m J_{m} & O & \cdots & O \\
O & m J_{m} & \cdots & O \\
\vdots & \vdots & \ddots & \vdots \\
O & O & \cdots & m J_{m}
\end{array}\right),
$$

which has only two distinct entries off diagonal. Therefore $M$ is a balancedly splittable Hadamard matrix of order $m^{2}$ with the parameters $\left(m^{2}, m, m, 0\right)$.

\subsection{Construction for $(n, \ell, a, b)=(k m, k(m-1), 0,-k), k, m$ orders for Hadamard matrices}

Theorem 3.3. Let $k, m$ be the orders for Hadamard matrices. Then there exists a balancedly splittable Hadamard matrix of order $k m$ with the parameters $(k m, k(m-1), 0,-k)$.

Proof. Let $H, K$ be Hadamard matrices of order $k, m$ respectively. Normalize $K$ so that $K=$ $\left(\begin{array}{l}\mathbf{1}^{\top} \\ K_{1}\end{array}\right)$. Then $H \otimes K$ is a Hadamard matrix and has $H \otimes K_{1}$ as a submatrix. Then using the property that $K_{1}^{\top} K_{1}=m I_{m}-J_{m}$, we have

$$
\left(H \otimes K_{1}\right)^{\top}\left(H \otimes K_{1}\right)=H^{\top} H \otimes K_{1}^{\top} K_{1}=k I_{k} \otimes\left(m I_{m}-J_{m}\right),
$$

which has only two distinct entries off diagonal. Therefore $H \otimes K$ is a balancedly splittable Hadamard matrix of order $k m$ with the parameters $(k m, k(m-1), 0,-k)$.

\subsection{Construction for $(n, \ell, a, b)=(n, n-2,0,-2), n$ an order for a Hadamard matrix}

The following result is the same as [2, Observation 2].

Theorem 3.4. Let $n$ be the order for a Hadamard matrix. Then there exists a balancedly splittable Hadamard matrix of order $n$ with the parameters $(n, n-2,0,-2)$.

Proof. Let $H$ be a Hadamard matrix of order $n$. Normalize the first two rows of $H$ so that $H=\left(\begin{array}{cc}\mathbf{1}_{n / 2}^{\top} & \mathbf{1}_{n / 2}^{\top} \\ \mathbf{1}_{n / 2}^{\top} & -\mathbf{1}_{n / 2}^{\top} \\ H_{1}\end{array}\right)$. Then $H_{1}^{\top} H_{1}=\left(\begin{array}{cc}n I_{n / 2}-2 J_{n / 2} & O_{n / 2} \\ O_{n / 2} & n I_{n / 2}-2 J_{n / 2}\end{array}\right)$, which has only two distinct entries off diagonal, where $O_{n / 2}$ denotes the zero matrix of order $n / 2$. It follows that $H$ is a balancedly splittable Hadamard matrix of order $n^{2}$ with the parameters $(n, n-2,0,-2)$.

3.5 Construction for $(n, \ell, a, b)=\left(4^{m}, 2^{m}, 2^{m}, 0\right),\left(4^{m}, 2^{m-1}\left(2^{m}-1\right), 2^{m-1},-2^{m-1}\right), m$ a positive integer

Let $H_{1}=\left(\begin{array}{cc}1 & 1 \\ 1 & -1\end{array}\right)$ and define $H_{m}=H_{m-1} \otimes H_{1}$ recursively for $m \geq 2$. Then $H_{m}$ is a Hadamard matrix of order $2^{m}$, which is called Sylvester-type. 
Lemma 3.5. If there exist a balancedly splittable Hadamard matrix of order $n_{i}^{2}$ with $\left(\ell_{i}, a_{i}, b_{i}\right)=$ $\left(n_{i}, n_{i}, 0\right)$ for $i=1,2$, then there exists a balancedly splittable Hadamard matrix $H$ of order $n_{1}^{2} n_{2}^{2}$ with $(\ell, a, b)=\left(n_{1} n_{2}, n_{1} n_{2}, 0\right)$.

Proof. Let $H_{i}=\left(\begin{array}{c}H_{i, 1} \\ H_{i, 2}\end{array}\right)$ be balancedly splittable Hadamard matrices of order $n_{i}^{2}$ with $\left(\ell_{i}, a_{i}, b_{i}\right)=$ $\left(n_{i}, n_{i}, 0\right)$ with respect to $H_{i, 1}$ for $i=1,2$. Then, by Remark [2.9, $H_{i, 1}^{\top} H_{i, 1}=n_{i} I_{n_{i}} \otimes J_{n_{i}}$ for $i=1,2$. A Hadamard matrix $H_{1} \otimes H_{2}$ has a submatrix $H_{1,1} \otimes H_{2,1}$. Then,

$$
\left(H_{1,1} \otimes H_{2,1}\right)^{\top}\left(H_{1,1} \otimes H_{2,1}\right)=H_{1,1}^{\top} H_{1,1} \otimes H_{2,1}^{\top} H_{2,1}=n_{1} n_{2} I_{n_{1}} \otimes J_{n_{1}} \otimes I_{n_{2}} \otimes J_{n_{2}},
$$

which is permutationally equal to $n_{1} n_{2} I_{n_{1} n_{2}} \otimes J_{n_{1} n_{2}}$. This proves that $H_{1} \otimes H_{2}$ is balancedly splittable.

Lemma 3.6. If there exist a balancedly splittable Hadamard matrix of order $n_{i}$ with $\left(\ell_{i}, a_{i}, b_{i}\right)=$ $\left(\left(n_{i}+\sqrt{n_{i}}\right) / 2, \sqrt{n_{i}} / 2,-\sqrt{n_{i}} / 2\right)$ for $i=1,2$, then there exists a balancedly splittable Hadamard matrix $H$ of order $n_{1} n_{2}$ with $(\ell, a, b)=\left(\left(n_{1} n_{2}+\sqrt{n_{1} n_{2}}\right) / 2, \sqrt{n_{1} n_{2}} / 2,-\sqrt{n_{1} n_{2}} / 2\right)$.

Proof. Let $H_{i}=\left(\begin{array}{l}H_{i, 1} \\ H_{i, 2}\end{array}\right)$ be balancedly splittable Hadamard matrices of order $n_{i}$ with $\left(\ell_{i}, a_{i}, b_{i}\right)=$ $\left(\left(n_{i}+\sqrt{n_{i}}\right) / 2, \sqrt{n_{i}} / 2,-\sqrt{n_{i}} / 2\right)$ for $i=1,2$. Then $H_{i, 1}^{\top} H_{i, 1}=\ell_{i} I_{n_{i}}+a_{i} S_{i}$ for some Seidel matrices $S_{i}, i=1,2$. We consider a Hadamard matrix $H_{1} \otimes H_{2}$, and it has $K:=\left(\begin{array}{l}H_{1,1} \otimes H_{2,1} \\ H_{1,2} \otimes H_{2,2}\end{array}\right)$ as a submatrix. Then

$$
\begin{aligned}
& K^{\top} K=\left(H_{1,1} \otimes H_{2,1}\right)^{\top}\left(H_{1,1} \otimes H_{2,1}\right)+\left(H_{1,2} \otimes H_{2,2}\right)^{\top}\left(H_{1,2} \otimes H_{2,2}\right) \\
& =H_{1,1}^{\top} H_{1,1} \otimes H_{2,1}^{\top} H_{2,1}+H_{1,2}^{\top} H_{1,2} \otimes H_{2,2}^{\top} H_{2,2} \\
& =\left(\ell_{1} I_{n_{1}}+a_{1} S_{1}\right) \otimes\left(\ell_{2} I_{n_{2}}+a_{2} S_{2}\right)+\left(\left(n_{1}-\ell_{1}\right) I_{n_{1}}-a_{1} S_{1}\right) \otimes\left(\left(n_{2}-\ell_{2}\right)-a_{2} S_{2}\right) \\
& =\left(\ell_{1} \ell_{2}+\left(n_{1}-\ell_{1}\right)\left(n_{2}-\ell_{2}\right)\right) I_{n_{1} n_{2}}+\left(2 \ell_{1}-n_{1}\right) a_{2} I_{n_{1}} \otimes S_{2}+a_{1}\left(2 \ell_{2}-n_{2}\right) S_{1} \otimes I_{n_{2}}+2 a_{1} a_{2} S_{1} \otimes S_{2} \\
& =\frac{n_{1} n_{2}+\sqrt{n_{1} n_{2}}}{2} I_{n_{1} n_{2}}+\frac{\sqrt{n_{1} n_{2}}}{2} I_{n_{1}} \otimes S_{2}+\frac{\sqrt{n_{1} n_{2}}}{2} S_{1} \otimes I_{n_{2}}+\frac{\sqrt{n_{1} n_{2}}}{2} S_{1} \otimes S_{2},
\end{aligned}
$$

which has only two distinct off-diagonal entires. Thus $H_{1} \otimes H_{2}$ is balancedly splittable.

A balancedly splittable Hadamard matrix $H$ of order $n^{2}$ is said to be twin if $H=\left(\begin{array}{l}H_{1} \\ H_{2} \\ H_{3}\end{array}\right)$ satisfies that $H$ is balancedly splittable with parameters $\left(n^{2}, n, n, 0\right)$ with respect to $H_{1}$ and with parameters $\left(n^{2}, n(n-1) / 2, n / 2,-n / 2\right)$ with respect to $H_{2}$ and $H_{3}$.

Theorem 3.7. The Sylvester-type Hadamard matrix of order $4^{m}$ is twin balancedly splittable.

Proof. Let $H$ be the Sylvester-type Hadamard matrix of order 4:

$$
H=\left(\begin{array}{l}
H_{1} \\
H_{2} \\
H_{3}
\end{array}\right)=\left(\begin{array}{cccc}
1 & 1 & 1 & 1 \\
1 & 1 & -1 & -1 \\
1 & -1 & 1 & -1 \\
1 & -1 & -1 & 1
\end{array}\right),
$$

where $H_{1}$ is a $2 \times 4$ matrix and $H_{2}, H_{3}$ are both $1 \times 4$ matrices. The result follows from Lemma 3.5 , Lemma 3.6 and the above Hadamard matrix of order 4 . 


\subsection{Construction for $(n, \ell, a, b)=(q(q+1), q, q,-1)$, where $q$ an order of a skew- symmetric Hadamard matrix}

In [27, it is shown that the existence of a skew-symmetric Hadamard matrix of order $q+1$ implies that the existence of a Hadamard matrix of order $(q-1) q$. We review the construction and its generalization.

In [7], the following matrices $\mathcal{J}_{m}^{(q)}$ and $\mathcal{A}_{m}^{(q)}$ are used in order to construct a quaternary complex Hadamard matrix. Let $q+1$ be the order of a skew type Hadamard matrix $H$. Multiply some rows and columns of $H$ by -1 , if necessary, we may assume that

$$
H=\left(\begin{array}{cc}
1 & \mathbf{1}^{\top} \\
-\mathbf{1} & I+Q
\end{array}\right)
$$

The $(0, \pm 1)$-matrix $Q=\left(q_{i j}\right)_{i, j=1}^{q}$, called the skew symmetric core of the skew type Hadamard matrix, is skew symmetric and satisfies that $J_{q} Q=Q J_{q}=O_{q}$, and $Q Q^{\top}=q I_{q}-J_{q}$. For any odd prime power $q$, see 21] for Paley's construction.

Let $q$ be the order of a skew symmetric core $Q$. Define the following matrices recursively for each nonnegative integer $m$ :

$$
\mathcal{J}_{m}^{(q)}=\left\{\begin{array}{ll}
J_{1} & \text { if } m=0, \\
J_{q} \otimes \mathcal{A}_{m-1}^{(q)} & \text { otherwise, }
\end{array} \quad \mathcal{A}_{m}^{(q)}= \begin{cases}J_{1} & \text { if } m=0, \\
I_{q} \otimes \mathcal{J}_{m-1}^{(q)}+Q \otimes \mathcal{A}_{m-1}^{(q)} & \text { otherwise. }\end{cases}\right.
$$

For a normalized Hadamard matrix of $H$ of order $q+1$ with skew symmetric core $Q$, the matrix $C=H-I$ is a conference matrix, that is, $C C^{\top}=q I$. We define $M=-I_{q+1} \otimes \mathcal{J}_{1}^{(q)}+C \otimes \mathcal{A}_{1}^{(q)}$.

Theorem 3.8. The matrix $M$ is a balancedly splittable Hadamard matrix of order $q(q+1)$ with $(n, \ell, a, b)=(q(q+1), q, q,-1)$.

Proof. To use the properties that $\mathcal{J}_{1}^{(q)}\left(\mathcal{J}_{1}^{(q)}\right)^{\top}+q \mathcal{A}_{1}^{(q)}\left(\mathcal{A}_{1}^{(q)}\right)^{\top}=q(q+1) I_{q}$ and $\mathcal{J}_{1}^{(q)}\left(\mathcal{A}_{1}^{(q)}\right)^{\top}=$ $\mathcal{A}_{1}^{(q)}\left(\mathcal{J}_{1}^{(q)}\right)^{\top}$, we have

$$
\begin{aligned}
M M^{\top} & =\left(-I_{q+1} \otimes \mathcal{J}_{1}^{(q)}+C \otimes \mathcal{A}_{1}^{(q)}\right)\left(-I_{q+1} \otimes\left(\mathcal{J}_{1}^{(q)}\right)^{\top}+C^{\top} \otimes\left(\mathcal{A}_{1}^{(q)}\right)^{\top}\right) \\
& =I_{q+1} \otimes \mathcal{J}_{1}^{(q)}\left(\mathcal{J}_{1}^{(q)}\right)^{\top}-C \otimes \mathcal{A}_{1}^{(q)}\left(\mathcal{J}_{1}^{(q)}\right)^{\top}-C^{\top} \otimes \mathcal{J}_{1}^{(q)}\left(\mathcal{A}_{1}^{(q)}\right)^{\top}+C C^{\top} \otimes \mathcal{A}_{1}^{(q)}\left(\mathcal{A}_{1}^{(q)}\right)^{\top} \\
& =I_{q+1} \otimes\left(\mathcal{J}_{1}^{(q)}\left(\mathcal{J}_{1}^{(q)}\right)^{\top}+q \mathcal{A}_{1}^{(q)}\left(\mathcal{A}_{1}^{(q)}\right)^{\top}\right)-C \otimes\left(\mathcal{A}_{1}^{(q)}\left(\mathcal{J}_{1}^{(q)}\right)^{\top}-\mathcal{J}_{1}^{(q)}\left(\mathcal{A}_{1}^{(q)}\right)^{\top}\right) \\
& =q(q+1) I_{q+1} \otimes I_{q} .
\end{aligned}
$$

Therefore $M$ is a Hadamard matrix. Next we show that $M$ is balancedly splittable with respect to $M_{1}$ obtained from $M$ by restricting rows to the first $q$ rows.

Since $M_{1}=\left(\begin{array}{llll}-\mathcal{J}_{1}^{(q)} & \mathcal{A}_{1}^{(q)} & \ldots & \mathcal{A}_{1}^{(q)}\end{array}\right)$ and

$$
\begin{aligned}
& \left(\mathcal{J}_{1}^{(q)}\right)^{\top} \mathcal{J}_{1}^{(q)}=\left(J_{q}\right)^{2}=q J_{q}, \\
& \left(\mathcal{J}_{1}^{(q)}\right)^{\top} \mathcal{A}_{1}^{(q)}=J_{q}\left(I_{q}+Q\right)=J_{q}, \\
& \left(\mathcal{A}_{1}^{(q)}\right)^{\top} \mathcal{A}_{1}^{(q)}=\left(I_{q}+Q^{\top}\right)\left(I_{q}+Q\right)=I_{q}+Q^{\top} Q=(q+1) I_{q}-J_{q},
\end{aligned}
$$

we have

$$
M_{1}^{\top} M_{1}=\left(\begin{array}{cccc}
q J_{q} & -J_{q} & \cdots & -J_{q} \\
-J_{q} & (q+1) I_{q}-J_{q} & \cdots & (q+1) I_{q}-J_{q} \\
\vdots & \vdots & \ddots & \vdots \\
-J_{q} & (q+1) I_{q}-J_{q} & \cdots & (q+1) I_{q}-J_{q}
\end{array}\right) .
$$

Thus $M$ is balancedly splittable. 


\section{Commutative association schemes}

In this section we define commutative association schemes.

A $d$-class commutative association scheme, see [1], with a finite vertex set $X$, is a set of non-zero $(0,1)$-matrices $A_{0}, A_{1}, \ldots, A_{d}$ with rows and columns indexed by $X$, such that

(1) $A_{0}=I_{|X|}$

(2) $\sum_{i=0}^{d} A_{i}=J_{|X|}$,

(3) $A_{i}^{\top} \in\left\{A_{1}, \ldots, A_{d}\right\}$ for any $i \in\{1, \ldots, d\}$,

(4) for all $i, j, A_{i} A_{j}=\sum_{k=0}^{d} p_{i, j}^{k} A_{k}$ for some non-negative integers $p_{i, j}^{k}$,

(5) for all $i, j, A_{i} A_{j}=A_{j} A_{i}$.

The association scheme is said to be symmetric if $A_{i}^{\top}=A_{i}$ for any $i$, and non-symmetric otherwise. Note that if symmetric matrices $A_{i}(0 \leq i \leq d)$ satisfy (4), then (5) must follow. The vector space spanned by $A_{i}$ 's over the real number field forms a commutative algebra, denoted by $\mathcal{A}$ and is called the Bose-Mesner algebra. Then there exists a basis of $\mathcal{A}$ consisting of primitive idempotents, say $E_{0}=(1 /|X|) J_{|X|}, E_{1}, \ldots, E_{d}$. Since $\left\{A_{0}, A_{1}, \ldots, A_{d}\right\}$ and $\left\{E_{0}, E_{1}, \ldots, E_{d}\right\}$ are two bases of $\mathcal{A}$, there exist the change-of-basis matrices $P=\left(P_{i, j}\right)_{i, j=0}^{d}, Q=\left(Q_{i, j}\right)_{i, j=0}^{d}$ so that

$$
A_{i}=\sum_{j=0}^{d} P_{j, i} E_{j}, \quad E_{j}=\frac{1}{|X|} \sum_{i=0}^{d} Q_{i, j} A_{i} .
$$

The matrices $P, Q$ are said to be the first and second eigenmatrices respectively.

Example 4.1. Construction in Subsection 3.5 is closely related to the binary Hamming schemes $H(n, 2)$. Let $X=\mathbb{Z}_{2}^{n}$ and $R_{i}=\{(x, y) \mid x, y \in X, d(x, y)=i\}$ for $i=0,1, \ldots, n$, where $d(x, y)$ is the Hamming distance between $x$ and $y$. Define $A_{i}$ to be the adjacency matrix of a graph $\left(X, R_{i}\right)$ for $i=0,1, \ldots, n$. Then the matrices $A_{0}, A_{1}, \ldots, A_{n}$ is a symmetric association scheme, which is called the binary Hamming association scheme, denoted by $H(n, 2)$.

We denote adjacency matrices of the Hamming scheme $H(n, 2)$ by $A_{i}^{(n)}$.

The Hamming scheme $H(n+1,2)$ is described as a fusion scheme of the product of schemes $H(n, 2)$ and $H(1,2)$ as follows, see also [3]. For association schemes $\left\{A_{0}^{\prime}, A_{1}^{\prime}, \ldots, A_{d_{1}}^{\prime}\right\}$ and $\left\{A_{0}^{\prime \prime}, A_{1}^{\prime \prime}, \ldots, A_{d_{2}}^{\prime \prime}\right\}$, the product of these two is an association schemes with non-zero matrices $(0,1) A_{i}^{\prime} \otimes A_{j}^{\prime \prime}$ where $0 \leq i \leq d_{1}, 0 \leq j \leq d_{2}$. Take two association schemes as the Hamming schemes $H(n, 2)$ and $H(1,2)$ respectively, then we have

$$
A_{i}^{(n+1)}=\sum_{j+k=i} A_{j}^{(n)} \otimes A_{k}^{(1)}=A_{i}^{(n)} \otimes A_{0}^{(1)}+A_{i-1}^{(n)} \otimes A_{1}^{(1)}
$$

for $i \in\{1, \ldots, n+1\}$. It follows now that the adjacency matrices of the binary Hamming scheme are diagonalizable by the Sylvester Hadamard matrices. For $n=1$, the adjacency matrices $A_{0}=I_{2}, A_{1}=J_{2}-I_{2}$ are diagonalizable by $H_{1}=\left(\begin{array}{cc}1 & 1 \\ 1 & -1\end{array}\right)$. For $n \geq 2$, it follows from the recurrence above that $H_{n}=H_{1}^{\otimes n}$ diagonalizes the adjacency matrices of $H(n, 2)$.

A subscheme or fusion scheme of the association scheme $\left(X,\left\{R_{i}\right\}_{i=0}^{d}\right)$ is an association scheme $\left(X,\left\{\cup_{j \in \Lambda_{i}} R_{j}\right\}_{i=0}^{e}\right)$ for some decomposition $\left\{\Lambda_{0}, \Lambda_{1}, \ldots, \Lambda_{e}\right\}$ of $\{0,1, \ldots, d\}$ such that $\Lambda_{0}=\{0\}$.

Muzychuk [20] classified the subschemes of $H(n, 2)$ for $n \geq 9$. By [20, Theorem 2.1], there are exactly two cases for the subschemes to be primitive strongly regular graphs: 
- $n$ is even and $\Lambda_{1}=\{k \mid 1 \leq k \leq n, k \equiv 0,1(\bmod 4)\}, \Lambda_{2}=\{k \mid 1 \leq k \leq n, k \equiv 2,3$ $(\bmod 4)\}$,

- $n$ is even and $\Lambda_{1}=\{k \mid 1 \leq k \leq n, k \equiv 0,3(\bmod 4)\}, \Lambda_{2}=\{k \mid 1 \leq k \leq n, k \equiv 1,2$ $(\bmod 4)\}$.

The parameters of these strongly regular graphs are

$$
(n, k, \lambda, \mu)=\left(4^{m}, 2^{m-1}\left(2^{m} \pm 1\right), 2^{m-1}\left(2^{m-1} \pm 1\right), 2^{m-1}\left(2^{m-1} \pm 1\right)\right)
$$

and their complements.

The Doob schemes are the association schemes with the same parameters as the binary Hamming schemes [6]. By Example 2.19, the Doob schemes are Hadamard diagonalizable, and this scheme has the fusion schemes which yield strongly regular graphs.

\section{Construction of commutative association schemes}

Let $H$ be a Hadamard matrix of order $n$ with rows $r_{1}, \ldots, r_{n}$. For $i \in\{1, \ldots, n\}$, let $C_{i}=r_{i}^{\top} r_{i}$. We call $C_{1}, \ldots, C_{n}$ the auxiliary matrices of $H$. The auxiliary matrices play an important role to construct association schemes. The following is basic properties for the auxiliary matrices.

Lemma 5.1. 14

(1) $\sum_{i=1}^{n} C_{i}=n I_{n}$.

(2) For any $i \in\{1, \ldots, n\}, C_{i}^{2}=n C_{i}$.

(3) For any distinct $i, j \in\{1, \ldots, n\}, C_{i} C_{j}=O_{n}$.

Note that for a Hadamard matrix $H$, letting $H=\left(\begin{array}{l}H_{1} \\ H_{2}\end{array}\right)$ where $H_{1}$ is an $\ell \times n$ matrix, it holds that $\sum_{i=1}^{\ell} C_{i}=H_{1}^{\top} H_{1}$.

Some combinatorial objects and association schemes are obtained from a balancedly splittable Hadamard matrix of order $n$ such that $\sum_{i=1}^{\ell} C_{i}$ has exactly one off-diagonal entries and some Latin squares as follows:

- symmetric or skew-symmetric Bush type Hadamard matrices and 3-class association schemes from the case $(\ell, a)=(n, 0)$ with $C_{1}=J_{n}$ and a symmetric Latin squares with constant diagonal, as described in [25], [9].

- symmetric or skew-symmetric regular $\left(0, \frac{1}{n-1}\right)$ biangular matrices and 4-class association schemes from the case $(\ell, a)=(n-1,1)$ with $C_{1}=J_{n}$ and a symmetric Latin square with constant diagonal, refer to [16].

- unbiased Hadamard matrices and 4-class association schemes from the case $(\ell, a)=(n, 0)$ and mutually unique fixed symbol (UFS) Latin squares, see [11, 19].

- unbiased Bush-type Hadamard matrices and 5-class association schemes from the case $(\ell, a)=(n, 0)$ with $C_{1}=J_{n}$ and mutually UFS Latin squares as defined in [15].

- unbiased biangular vectors (more generally linked systems of symmetric group divisible designs) and 5-class association schemes from the case $(\ell, a)=(n-1,1)$ with $C_{1}=J_{n}$ and mutually UFS Latin squares, as shown in [13], and [17]. 
In the following subsections, we construct symmetric or non-symmetric association schemes with 4,5 or 6 -classes from a balancedly splittable Hadamard matrix such that $\sum_{i=1}^{\ell} C_{i}$ has exactly two distinct off diagonal entries and some Latin squares. Throughout the following subsections, we assume that $H$ is a balancedly splittable Hadamard matrix of order $n$ with auxiliary matrices $C_{1}, \ldots, C_{n}$ satisfying $\sum_{i=1}^{\ell} C_{i}=\ell I_{n}+a A+b\left(J_{n}-A-I_{n}\right)$ where $A$ is an $n \times n(0,1)$-matrix, $a \neq b$, and $C_{i} J_{n}=O_{n}$ for $i \in\{1, \ldots, \ell\}$. According to Proposition 2.6. $b=\frac{\ell(-a+\ell-n)}{a(n-1)+\ell}$ and the matrix $A$ is the adjacency matrix of a strongly regular graph with the parameters $(n, k, \lambda, \mu)$ given as:

$$
\begin{aligned}
k & =\frac{\ln (n-\ell-1)}{n\left(a^{2}+\ell\right)-(a-\ell)^{2}}, \\
\lambda & =\frac{n\left(n^{2}\left(a^{3}+\ell^{2}\right)-2(\ell+1) n\left(a^{3}+\ell^{2}\right)+(2 a \ell+a+\ell(\ell+2))(a-\ell)^{2}\right)}{\left((a-\ell)^{2}-n\left(a^{2}+\ell\right)\right)^{2}}, \\
\mu & =\frac{\ln (a-\ell)(\ell-n+1)(a-\ell+n)}{\left((a-\ell)^{2}-n\left(a^{2}+\ell\right)\right)^{2}} .
\end{aligned}
$$

We use $C_{0}:=O_{n}$ and a Latin square $L=\left(L_{i, j}\right)_{i, j \in S}$ on the symbol set $S$ where $S$ equals to $\{1, \ldots, \ell\}$ or $\{0,1, \ldots, \ell\}$, and denote $\tilde{L}$ to be

$$
\tilde{L}=\left(C_{L_{i, j}}\right)_{i, j \in S}
$$

For the remaining part of the paper, we use a variant of Mutually Orthogonal Latin Squares $(M O L S)$ which we call UFS (Unique Fix Symbol) suitable for the way we apply it. Two Latin squares $L_{1}$ and $L_{2}$ of size $n$ on the same symbol set are called to be UFS Latin squares, if every superimposition of each row of $L_{1}$ on each row of $L_{2}$ results in only one element of the form $(a, a)$. In effect, each permutation of symbols between the rows of the two Latin squares has a Unique Fixed Symbol. A set of Latin squares in which every distinct pair of Latin squares are UFS Latin square is called mutually UFS Latin squares. Note that UFS Latin squares are called suitable Latin squares in [11] and elsewhere. See [11] for the equivalentness of existence between mutually UFS Latin squares and mutually orthogonal Latin squares.

Lemma 5.2. Let $L_{1}, L_{2}$ be UFS Latin squares on the symbol set $\{1, \ldots, n\}$ with the $(i, j)$-entry equal to $l(i, j), l^{\prime}(i, j)$ respectively. An $n \times n$ array with the $(i, j)$-entry equal to $b$ determined by $b=l(i, a)=l^{\prime}(j, a)$ for the unique $a \in\{1, \ldots, n\}$, is a Latin square.

The following lemma will be used in Subsections 5.1, 5.2, 5.3, We omit its easy proof.

Lemma 5.3. Let $H$ be a balancedly splittable Hadamard matrix of order $n$. If $C_{i} J_{n}=J_{n} C_{i}=$ $O_{n}$, then $A C_{i}=C_{i} A=(n-\ell+b) C_{i}$.

Lemma 5.4. Let $H$ be a balancedly splittable Hadamard matrix of order $n$ with $\sum_{i=1}^{\ell} C_{i}=\ell I_{n}+$ $a A+b\left(J_{n}-A-I_{n}\right)$ and $C_{i} J_{n}=O_{n}$ for $i \in\{1, \ldots, \ell\}$. Let $L$ be a Latin square on the symbol set $S$ where $S$ equals to $\{1, \ldots, \ell\}$ or $\{0,1, \ldots, \ell\}$. Then $\tilde{L} \tilde{L}^{\top}=n I_{|S|} \otimes\left(|S| I_{n}+a A+b\left(J_{n}-A-I_{n}\right)\right)$.

Lemma 5.5. Let $H$ be a balancedly splittable Hadamard matrix of order $n$ with $\sum_{i=1}^{\ell} C_{i}=$ $\ell I_{n}+a A+b\left(J_{n}-A-I_{n}\right)$ and $C_{i} J_{n}=O_{n}$ for $i \in\{1, \ldots, \ell\}$. Let $L_{1}, \ldots, L_{f}$ be mutually UFS Latin squares on the symbol set $S$ where $S$ equals to $\{1, \ldots, \ell\}$ or $\{0,1, \ldots, \ell\}$. For distinct $i, j \in$ $\{1, \ldots, f\}, \tilde{L}_{i} \tilde{L}_{j}^{\top}=n \tilde{L}_{i, j}$, where $L_{i, j}$ is the Latin square determined from $L_{1}, L_{2}$ by Lemma [5.2.

Then the following holds: for any distinct $i, j, k \in\{1, \ldots, f\}, L_{i, k}$ and $L_{j, k}$ are UFS and the Latin square obtained from $L_{i, k}$ and $L_{j, k}$ in this ordering via Lemma 5.2 coincides with $L_{i, j}$. 


\subsection{Symmetric and non-symmetric association schemes with 4-classes}

In this subsection, we will use a symmetric Latin square with constant diagonal, which is known to exist for order $v$ a positive even integer, see [14. Assume that $\ell$ is an odd integer and let $L$ be a symmetric Latin square of order $\ell+1$ on the symbol set $\{0,1, \ldots, \ell\}$ with constant diagonal 0 .

We define disjoint $(0,1)$-matrices $A_{i}(i \in\{0,1, \ldots, 4\})$ as

$$
A_{0}=I_{(\ell+1) n}, \quad A_{1}=I_{\ell+1} \otimes A, \quad A_{2}=I_{\ell+1} \otimes\left(J_{n}-A-I_{n}\right), \quad \tilde{L}=A_{3}-A_{4} .
$$

Theorem 5.6. The set of matrices $\left\{A_{0}, A_{1}, \ldots, A_{4}\right\}$ is a symmetric association scheme with 4-classes.

Proof. It is routine to see that $A_{0}=I_{(\ell+1) n}, A_{i}$ 's are disjoint symmetric $(0,1)$-matrices such that $\sum_{i=0}^{4} A_{i}=J_{(\ell+1) n}$, and each $A_{i}$ is symmetric. Let $\mathcal{A}=\operatorname{span}_{\mathbb{R}}\left\{A_{0}, A_{1}, \ldots, A_{4}\right\}$. We will check (4) in the definition of the association scheme for each case.

(i): For $i, j \in\{1,2\}$, (AS4) follows from the fact that $A$ is the adjacency matrix of a strongly regular graph.

(ii): It follows from Lemma 5.3 that $A_{i}\left(A_{3}-A_{4}\right),\left(A_{3}-A_{4}\right) A_{i} \in \mathcal{A}$ for $i=1,2$. Since $A_{3}+A_{4}=I_{\ell+1} \otimes\left(J_{n}-I_{n}\right)$ and $A$ is in particular the adjacency matrix of a regular graph, $A_{i}\left(A_{3}+A_{4}\right),\left(A_{3}+A_{4}\right) A_{i} \in \mathcal{A}$ for $i=1,2$. Thus (AS4) holds for $(i, j) \in(\{1,2\} \times\{3,4\}) \cup$ $(\{3,4\} \times\{1,2\})$.

(iii): For $i, j \in\{3,4\},\left(A_{3}-A_{4}\right)^{2}=(\tilde{L})^{2} \in \mathcal{A}$ by Lemma 5.4. By $A_{3}+A_{4}=\left(J_{\ell+1}-I_{\ell+1}\right) \otimes J_{n}$ and $C_{i} J_{n}=O_{n}$ for $i \in\{1, \ldots, \ell\},\left(A_{3}+A_{4}\right)\left(A_{3}-A_{4}\right)=\left(A_{3}-A_{4}\right)\left(A_{3}+A_{4}\right)=O_{n} \in \mathcal{A}$ and $\left(A_{3}+A_{4}\right)^{2} \in \mathcal{A}$. These implies that each component of $\left(A_{3}^{2}, A_{3} A_{4}, A_{4} A_{3}, A_{4}^{2}\right) H$ belongs to $\mathcal{A}$ where $H$ is a Hadamard matrix. Since $H$ is invertible, each of $A_{3}^{2}, A_{3} A_{4}, A_{4} A_{3}, A_{4}^{2}$ belongs to $\mathcal{A}$.

This completes the proof.

Then the eigenmatrices $P$ and $Q$ are as follows:

$$
\begin{aligned}
& P=\left(\begin{array}{cccccc}
1 & \frac{\ell(n-\ell-1) n}{(n-1) a^{2}+2 \ell a+\ell(n-\ell)} & \frac{(\ell+a(n-1))^{2}}{(n-1) a^{2}+2 \ell a+\ell(n-\ell)} & \frac{\ell n}{2} & \frac{\ell n}{2} \\
1 & \frac{\ell(n-\ell-1) n}{(n-1) a^{2}+2 \ell a+\ell(n-\ell)} & \frac{(\ell+a(n-1))^{2}}{(n-1) a^{2}+2 \ell a+\ell(n-\ell)} & -\frac{n}{2} & -\frac{n}{2} \\
1 & \frac{a(n-\ell-1) n}{(n-1) a^{2}+2 \ell a+\ell(n-\ell)} & \frac{(\ell+a(n-1))(n+a-\ell)}{(n-1) a^{2}+2 \ell a+\ell(n-\ell)} & -\frac{n}{2} & \frac{n}{2} \\
1 & \frac{a(n-\ell-1) n}{(n-1) a^{2}+2 \ell a+\ell(n-\ell)} & \frac{(\ell+a(n-1))(n+a-\ell)}{(n-1) a^{2}+2 \ell a+\ell(n-\ell)} & \frac{n}{2} & -\frac{n}{2} \\
1 & -\frac{(a+1) \ell n}{(n-1) a^{2}+2 \ell a+\ell(n-\ell)} & -\frac{(a-\ell)(\ell+a(n-1))}{(n-1) a^{2}+2 \ell a+\ell(n-\ell)} & 0 & 0
\end{array}\right), \\
& Q=\left(\begin{array}{ccccc}
1 & \ell & \frac{1}{2} \ell(\ell+1) & \frac{1}{2} \ell(\ell+1) & (\ell+1)(n-\ell-1) \\
1 & \ell & \frac{1}{2} a(\ell+1) & \frac{1}{2} a(\ell+1) & -(a+1)(\ell+1) \\
1 & \ell & \frac{\ell(\ell+1)(\ell-n-a)}{2(\ell+a(n-1))} & \frac{\ell(\ell+1)(\ell-n-a)}{2(\ell+a(n-1))} & \frac{(\ell-a)(\ell+1)(n-\ell-1)}{\ell+a(n-1)} \\
1 & -1 & \frac{-\ell-1}{2} & \frac{\ell+1}{2} & 0 \\
1 & -1 & \frac{\ell+1}{2} & \frac{-\ell-1}{2} & 0
\end{array}\right) .
\end{aligned}
$$

By a slight modification, we obtain non-symmetric association schemes with 4-classes. Under the same setting on $L, \ell$, and $C_{i}$ as above, we define $\bar{L}=\left(\epsilon_{i, j} C_{L_{i, j}}\right)_{i, j=1}^{\ell+1}$, where $\epsilon_{i, j}=1$ if $i \leq j$ and -1 if $i>j$. We define disjoint $(0,1)$-matrices $A_{i}(i \in\{0,1, \ldots, 4\})$ as

$$
A_{0}=I_{(\ell+1) n}, \quad A_{1}=I_{\ell+1} \otimes A, \quad A_{2}=I_{\ell+1} \otimes\left(J_{n}-A-I_{n}\right), \quad \bar{L}=A_{3}-A_{4} .
$$

Note that $A_{3}^{\top}=A_{4}$.

Theorem 5.7. The set of matrices $\left\{A_{0}, A_{1}, \ldots, A_{4}\right\}$ is a non-symmetric association scheme with 4-classes. 
Proof. The proof is the same as that of Theorem 5.6.

The eigenmatrices $\tilde{P}$ and $\tilde{Q}$ are obtained from $P, Q$ by changing $\tilde{P}_{i, j}=\sqrt{-1} P_{i, j}$ for $i \in$ $\{2,3\}, j \in\{3,4\}$ and $\tilde{Q}_{i, j}=\sqrt{-1} Q_{i, j}$ for $i \in\{3,4\}, j \in\{2,3\}$.

\subsection{Symmetric association schemes with 5-classes}

Let $L_{1}, \ldots, L_{f}$ be mutually UFS Latin squares on $\{1, \ldots, \ell\}$. We now construct a symmetric association scheme with 5-classes from a balancedly splittable Hadamard matrix and mutually UFS Latin squares. Consider the Gram matrix $G$ of the row vectors of $\tilde{L}_{i}(i \in\{1, \ldots, f\})$ defined by

$$
G=\left(\begin{array}{cccc}
\tilde{L}_{1} \tilde{L}_{1}^{\top} & \tilde{L}_{1} \tilde{L}_{2}^{\top} & \cdots & \tilde{L}_{1} \tilde{L}_{f}^{\top} \\
\tilde{L}_{2} \tilde{L}_{1}^{\top} & \tilde{L}_{2} \tilde{L}_{2}^{\top} & \cdots & \tilde{L}_{2} \tilde{L}_{f}^{\top} \\
\vdots & \vdots & \ddots & \vdots \\
\tilde{L}_{f} \tilde{L}_{1}^{\top} & \tilde{L}_{f} \tilde{L}_{2}^{\top} & \cdots & \tilde{L}_{f} \tilde{L}_{f}^{\top}
\end{array}\right)
$$

The entries of $G$ are $\{n \ell, n a, n b, \pm n, 0\}$. Decompose the matrix $G$ into its entries as

$$
G=n \ell A_{0}+n\left(a A_{1}+b A_{2}\right)+n\left(A_{3}-A_{4}\right) .
$$

Then the disjoint $(0,1)$-matrices $A_{i}(i \in\{0,1, \ldots, 4\})$ satisfy $\sum_{i=0}^{4} A_{i}=J_{f \ell n}-I_{f} \otimes\left(J_{\ell}-I_{\ell}\right) \otimes J_{n}$. We now define

$$
A_{5}=I_{f} \otimes\left(J_{\ell}-I_{\ell}\right) \otimes J_{n} .
$$

Note that $A_{1}=I_{f} \otimes I_{\ell} \otimes A, A_{2}=I_{f} \otimes I_{\ell} \otimes\left(J_{n}-A-I_{n}\right)$ and

$$
\begin{aligned}
& A_{3}-A_{4}=\frac{1}{n}\left(\begin{array}{cccc}
O_{\ell n} & \tilde{L}_{1} \tilde{L}_{2}^{\top} & \cdots & \tilde{L}_{1} \tilde{L}_{f}^{\top} \\
\tilde{L}_{2} \tilde{L}_{1}^{\top} & O_{\ell n} & \cdots & \tilde{L}_{2} \tilde{L}_{f}^{\top} \\
\vdots & \vdots & \ddots & \vdots \\
\tilde{L}_{f} \tilde{L}_{1}^{\top} & \tilde{L}_{f} \tilde{L}_{2}^{\top} & \cdots & O_{\ell n}
\end{array}\right), \\
& A_{3}+A_{4}=\left(J_{f}-I_{f}\right) \otimes J_{\ell} \otimes J_{n} .
\end{aligned}
$$

The following is the main result of this subsection.

Theorem 5.8. Let $H$ be a balancedly splittable Hadamard matrix of order $n$ with $\sum_{i=1}^{\ell} C_{i}=$ $\ell I_{n}+a A+b\left(J_{n}-A-I_{n}\right)$ where $A$ is the adjacency matrix of a regular graph, and $L_{1}, \ldots, L_{f}$ be mutually UFS Latin square on $\{1, \ldots, \ell\}$. Then the set of matrices $\left\{A_{0}, A_{1}, \ldots, A_{5}\right\}$ defined above is a symmetric association scheme with 5-classes.

Proof. It is easy to see that $A_{0}=I_{f \ell_{n}}, A_{i}$ 's are disjoint symmetric $(0,1)$-matrices such that $\sum_{i=0}^{5} A_{i}=J_{\text {f } n}$, and each $A_{i}$ is symmetric. Let $\mathcal{A}=\operatorname{span}_{\mathbb{R}}\left\{A_{0}, A_{1}, \ldots, A_{5}\right\}$.

First it can be shown that $\operatorname{span}_{\mathbb{R}}\left\{A_{0}, A_{1}, A_{2}, A_{3}+A_{4}, A_{5}\right\}$ is closed under the matrix multiplication. Next we show that the products $A_{i}\left(A_{3}-A_{4}\right)$ for $i \in\{1,2,5\}$ and $\left(A_{3}-A_{4}\right)^{2}$ are linear combinations of $A_{0}, A_{1}, \ldots, A_{5}$, from which (4) in the definition of the association scheme follows. The equation $A_{5}\left(A_{3}-A_{4}\right)=O_{f \ell n}$ can be shown, and the cases for $A_{i}\left(A_{3}-A_{4}\right)$ for $i \in\{1,2\}$ follow from the following.

Since $\left(I_{\ell} \otimes J_{n}\right) \tilde{L}_{j}=O_{\ell n}$ for each $j$, we have that $\left(A_{0}+A_{1}+A_{2}\right)\left(A_{3}-A_{4}\right)=O_{f \ell n}$. Therefore $\left(A_{1}+A_{2}\right)\left(A_{3}-A_{4}\right)=-A_{3}+A_{4}$.

Since $\left(\sum_{i=1}^{\ell} I_{\ell} \otimes C_{i}\right) \tilde{L}_{j}=\tilde{L}_{j}$ for each $j$, we have that $\left(a A_{1}+b A_{2}\right)\left(A_{3}-A_{4}\right)=(n-\ell)\left(A_{3}-A_{4}\right)$. 
Finally from Lemmas 5.4, 5.5] it follows that

$$
\left(A_{3}-A_{4}\right)^{2}=n(f-1)\left(\ell A_{0}+a A_{1}+b A_{2}\right)+n(f-2)\left(A_{3}-A_{4}\right) .
$$

This completes the proof.

Then the eigenmatrices $P$ and $Q$ are as follows:

$$
\begin{aligned}
& P=\left(\begin{array}{ccccccc}
1 & \frac{\ell(n-\ell-1) n}{(n-1) a^{2}+2 \ell a+\ell(n-\ell)} & \frac{(\ell+a(n-1))^{2}}{(n-1) a^{2}+2 \ell a+\ell(n-\ell)} & \frac{1}{2}(f-1) \ell n & \frac{1}{2}(f-1) \ell n & (\ell-1) n \\
1 & \frac{a n(n-\ell-1)}{(n-1) a^{2}+2 \ell a+\ell(n-\ell)} & -\frac{(\ell+a(n-1))(a-\ell+n)}{(n-1) a^{2}+2 l a+\ell(n-\ell)} & \frac{1}{2}(f-1) n & \frac{1}{2}(n-f n) & 0 \\
1 & -\frac{(a+1) \ell n}{(n-1) a^{2}+2 \ell a+\ell(n-\ell)} & -\frac{(a-\ell)(\ell+a(n-1))}{(n-1) a^{2}+2 \ell a+\ell(n-\ell)} & 0 & 0 & 0 \\
1 & \frac{\ell(\ell-n+1) n}{-(n-1) a^{2}-2 \ell a+\ell(\ell-n)} & \frac{(\ell+a(n-1))^{2}}{(n-1) a^{2}+2 \ell a+\ell(n-\ell)} & 0 & 0 & -n \\
1 & \frac{a n(-\ell+n-1)}{(n-1) a^{2}+2 \ell a+\ell(n-\ell)} & -\frac{(\ell+a(n-1))(a-\ell+n)}{(n-1) a^{2}+2 \ell a+\ell(n-\ell)} & -\frac{n}{2} & \frac{n}{2} & 0 \\
1 & \frac{\ell(\ell-n+1) n}{-(n-1) a^{2}-2 \ell a+\ell(\ell-n)} & \frac{(\ell+a(n-1))^{2}}{(n-1) a^{2}+2 \ell a+\ell(n-\ell)} & -\frac{1}{2} \ell n & -\frac{1}{2} \ell n & (\ell-1) n
\end{array}\right), \\
& Q=\left(\begin{array}{cccccc}
1 & \ell^{2} & f \ell(n-\ell-1) & f(\ell-1) & (f-1) \ell^{2} & f-1 \\
1 & a \ell & -(a+1) f \ell & f(\ell-1) & a(f-1) \ell & f-1 \\
1 & \frac{\ell^{2}(-a+\ell-n)}{\ell+a(n-1)} & \frac{f(a-\ell) \ell(\ell-n+1)}{\ell+a(n-1)} & f(\ell-1) & -\frac{(f-1) \ell^{2}(a-\ell+n)}{\ell+a(n-1)} & f-1 \\
1 & \ell & 0 & 0 & -\ell & -1 \\
1 & -\ell & 0 & 0 & \ell & -1 \\
1 & 0 & 0 & -f & 0 & f-1
\end{array}\right) .
\end{aligned}
$$

\subsection{Symmetric association schemes with 6-classes}

Let $L_{1}, \ldots, L_{f}$ be mutually UFS Latin squares on $\{0,1, \ldots, \ell\}$ with constant diagonal 0 . We now construct a symmetric association scheme with 6-classes from a balancedly splittable Hadamard matrix and mutually UFS Latin squares. Consider the Gram matrix $G$ of the row vectors of $\tilde{L}_{i}$ $(i \in\{1, \ldots, f\})$ defined by $G=\left(\tilde{L}_{i} \tilde{L}_{j}^{\top}\right)_{i, j=1}^{f}$

The entries of $G$ are $\{n \ell, n a, n b, \pm n, 0\}$. Decompose the matrix $G$ into its entries as

$$
G=n \ell A_{0}+n\left(a A_{1}+b A_{2}\right)+n\left(A_{3}-A_{4}\right) .
$$

Then the disjoint $(0,1)$-matrices $A_{i}(i \in\{0,1, \ldots, 4\})$ satisfy $\sum_{i=0}^{4} A_{i}=J_{f(\ell+1) n}-\left(I_{f} \otimes\left(J_{\ell+1}-\right.\right.$ $\left.\left.I_{\ell+1}\right) \otimes J_{n}+\left(J_{f}-I_{f}\right) \otimes I_{\ell+1} \otimes J_{n}\right)$. We now define

$$
A_{5}=I_{f} \otimes\left(J_{\ell+1}-I_{\ell+1}\right) \otimes J_{n}, \quad A_{6}=\left(J_{f}-I_{f}\right) \otimes I_{\ell+1} \otimes J_{n} .
$$

Note that $A_{1}=I_{f} \otimes I_{\ell+1} \otimes A, A_{2}=I_{f} \otimes I_{\ell+1} \otimes\left(J_{n}-A-I_{n}\right)$ and

$$
\begin{gathered}
A_{3}-A_{4}=\frac{1}{n}\left(\begin{array}{cccc}
O_{(\ell+1) n} & \tilde{L}_{1} \tilde{L}_{2}^{\top} & \cdots & \tilde{L}_{1} \tilde{L}_{f}^{\top} \\
\tilde{L}_{2} \tilde{L}_{1}^{\top} & O_{(\ell+1) n} & \cdots & \tilde{L}_{2} \tilde{L}_{f}^{\top} \\
\vdots & \vdots & \ddots & \vdots \\
\tilde{L}_{f} \tilde{L}_{1}^{\top} & \tilde{L}_{f} \tilde{L}_{2}^{\top} & \cdots & O_{(\ell+1) n}
\end{array}\right), \\
A_{3}+A_{4}=\left(J_{f}-I_{f}\right) \otimes\left(J_{\ell+1}-I_{\ell+1}\right) \otimes J_{n} .
\end{gathered}
$$

The following is the main result of this subsection.

Theorem 5.9. Let $H$ be a balancedly splittable Hadamard matrix of order $n$ with $\sum_{i=1}^{\ell} C_{i}=$ $\ell I_{n}+a A+b\left(J_{n}-A-I_{n}\right)$ where $A$ is the adjacency matrix of a regular graph, and $L_{1}, \ldots, L_{f}$ be mutually UFS Latin square on $\{0,1, \ldots, \ell\}$ with constant diagonal 0 . Then the set of matrices $\left\{A_{0}, A_{1}, \ldots, A_{6}\right\}$ defined above is a symmetric association scheme with 6-classes. 
Proof. The proof is similar to that of Theorem 5.8 .

Then the eigenmatrices $P$ and $Q$ are as follows:

$$
\begin{aligned}
P & =\left(\begin{array}{ccccccc}
1 & \frac{\ell(\ell-n+1) n}{-(n-1) a^{2}-2 \ell a+\ell(\ell-n)} & \frac{(\ell+a(n-1))^{2}}{(n-1) a^{2}+2 \ell a+\ell(n-\ell)} & \frac{1}{2}(f-1) \ell n & \frac{1}{2}(f-1) \ell n & \ell n & (f-1) n \\
1 & \frac{\ell(\ell-n+1) n}{-(n-1) a^{2}-2 \ell a+\ell(\ell-n)} & \frac{(\ell+a(n-1))^{2}}{(n-1) a^{2}+2 \ell a+\ell(n-\ell)} & -\frac{1}{2}(f-1) n & -\frac{1}{2}(f-1) n & -n & (f-1) n \\
1 & \frac{a n(-\ell+n-1)}{(n-1) a^{2}+2 \ell a+\ell(n-\ell)} & -\frac{(\ell+a(n-1))(a-\ell+n)}{(n-1) a^{2}+2 \ell a+\ell(n-\ell)} & \frac{1}{2}(f-1) n & \frac{1}{2}(n-f n) & 0 & 0 \\
1 & -\frac{(a+1) \ell n}{(n-1) a^{2}+2 \ell a+\ell(n-\ell)} & -\frac{(a-\ell)(\ell+a(n-1))}{(n-1) a^{2}+2 \ell a+\ell(n-\ell)} & 0 & 0 & 0 & 0 \\
1 & \frac{a n(-\ell+n-1)}{(n-1) a^{2}+2 \ell a+\ell(n-\ell)} & -\frac{(\ell+a(n-1))(a-\ell+n)}{(n-1) a^{2}+2 \ell a+\ell(n-\ell)} & -\frac{n}{2} & \frac{n}{2} & 0 & 0 \\
1 & \frac{\ell(\ell-n+1) n}{-(n-1) a^{2}-2 \ell a+\ell(\ell-n)} & \frac{(\ell+a(n-1))^{2}}{(n-1) a^{2}+2 \ell a+\ell(n-\ell)} & \frac{n}{2} & \frac{n}{2} & -n & -n \\
1 & \frac{\ell(\ell-n+1) n}{-(n-1) a^{2}-2 \ell a+\ell(\ell-n)} & \frac{(\ell+a(n-1))^{2}}{(n-1) a^{2}+2 \ell a+\ell(n-\ell)} & -\frac{1}{2}(\ell n) & -\frac{1}{2}(\ell n) & \ell n & -n
\end{array}\right), \\
Q= & \left(\begin{array}{ccccccc}
1 & \ell & \ell(\ell+1) & -f(\ell+1)(\ell-n+1) & (f-1) \ell(\ell+1) & (f-1) \ell & f-1 \\
1 & \ell & a(\ell+1) & -(a+1) f(\ell+1) & a(f-1)(\ell+1) & (f-1) \ell & f-1 \\
1 & \ell & \frac{\ell(\ell+1)(-a+\ell-n)}{\ell+a(n-1)} & \frac{f(a-\ell)(\ell+1)(\ell-n+1)}{\ell+a(n-1)} & -\frac{(f-1) \ell(\ell+1)(a-\ell+n)}{\ell+a(n-1)} & (f-1) \ell & f-1 \\
1 & -1 & \ell+1 & 0 & -\ell-1 & 1 & -1 \\
1 & -1 & -\ell-1 & 0 & \ell+1 & 1 & -1 \\
1 & -1 & 0 & 0 & 0 & 1-f & f-1 \\
1 & \ell & 0 & 0 & 0 & -l & -1
\end{array}\right) .
\end{aligned}
$$

\section{Acknowledgments.}

The authors are grateful to an anonymous referee for pointing out some calculation errors and for many suggestions which has improved the presentation of the paper. The authors acknowledge very useful conversation with Darcy Best. Hadi Kharaghani is supported by the Natural Sciences and Engineering Research Council of Canada (NSERC). Sho Suda is supported by JSPS KAKENHI Grant Number 15K21075 and 18K03395.

\section{References}

[1] E. Bannai, T. Ito, Algebraic Combinatorics I: Association Schemes, Benjamin/Cummings, Menlo Park, CA, 1984.

[2] S. Barik, S. Fallat, S. Kirkland, On Hadamard diagonalizable graphs, Linear Algebra and Appl. 435 (2011) 1885-1902.

[3] A. E. Brouwer, H. O. Hamalainen, P. R. J. Ostergard, N. J. A. Sloane, Bounds on mixed binary/ternary codes. IEEE Trans. Inform. Theory 44 (1998), no. 1, 140-161.

[4] A. E. Brouwer, W. H. Haemers, Spectra of graphs, Universitext. Springer, New York, 2012. xiv $+250 \mathrm{pp}$.

[5] P. Delsarte, J. M. Goethals, J. J. Seidel, Spherical codes and designs, Geom. Dedicata 6 (1977), 363-388.

[6] M. Doob, On graphs products and association schemes, Utilitas Math. 1 (1972), 291-302.

[7] K. Fender, H. Kharaghani, S. Suda On a class of quaternary complex Hadamard matrices, Discrete Math. 341 (2018), 421-426. 
[8] J. M. Goethals, J. J. Seidel, Strongly regular graphs derived from combinatorial designs, Canad. J. Math. 22 (1970), 597-614.

[9] R. W. Goldbach, H.L. Claasen, 3-class association schemes and Hadamard matrices of a certain block form, Europ. J. Combin. 19 (1998), 943-951.

[10] W. H. Holzmann, H. Kharaghani, Lavassani, M. T., The excess problem and some excess inequivalent matrices of order 32. R. C. Bose Memorial Conference (Fort Collins, CO, 1995). J. Statist. Plann. Inference 72 (1998), no. 1-2, 381-391.

[11] W. H. Holzmann, H. Kharaghani, W. Orrick, On the real unbiased Hadamard matrices. Combinatorics and graphs, 243-250, Contemp. Math., 531, Amer. Math. Soc., Providence, RI, 2010.

[12] W. H. Holzmann, H. Kharaghani, S. Sane, On a class of quasi-symmetric designs, J. Combin. Math. Combin. Comput. 57 (2006), 103-106.

[13] W. H. Holzmann, H. Kharaghani, S. Suda, Mutually unbiased biangular vectors and association schemes, In C. J. Colbourn, editor, Algebraic Design Theory and Hadamard Matrices, volume 133 of Springer Proceedings in Mathematics Statistics, pages 149-157. Springer International Publishing, 2015.

[14] H. Kharaghani, New class of weighing matrices, Ars. Combin. 19 (1985), 69-72.

[15] H. Kharaghani, S. Sasani, S. Suda, Mutually unbiased Bush-type Hadamard matrices and association schemes, Elec. J. Combin. 22 (2015), \#P3.10.

[16] H. Kharaghani, S. Suda, Hoffman's coclique bound for normal regular digraphs, and nonsymmetric association schemes, Mathematics Across Contemporary Sciences, 137-150, Springer Proc. Math. Stat., 190, Springer, Cham, 2017.

[17] H. Kharaghani, S. Suda, Linked systems of symmetric group divisible designs, J. Algebraic Combin. 47 (2017), no. 2, 319-343.

[18] H. Kharaghani, S. Suda, Linked system of symmetric group divisible designs of type II, submitted. [arXiv:1710:07888].

[19] N. LeCompte, W. J. Martin, W. Owens, On the equivalence between real mutually unbiased bases and a certain class of association schemes, Eur. J. Combin. 31 (2010), 1499-1512.

[20] M. E. Muzychuk, The subschemes of the Hamming scheme, Investigations in the algebraic theory of combinatorial objects, Mathematics and Its Applications, (1992), 187-208.

[21] R.E.A.C. Paley, On orthogonal matrices, J. Math. Phys. 12 (1933), 311-320.

[22] K. B. Reid and E. Brown, Doubly regular tournaments are equivalent to skew Hadamard matrices, J. Combin. Theory Ser. A 12 (1972), 332-338.

[23] S.S. Shrikhande, The uniqueness of the $L_{2}$ association scheme, The Annals of Mathematical Statistics, 30(3) (1959), 781-798.

[24] J. J. Seidel, Strongly regular graphs with $(-1,1,0)$ adjacency matrix having eigenvalue 3. Linear Algebra and Appl. 1 (1968) 281-298.

[25] W. D. Wallis, On a problem of K. A. Bush concerning Hadamard matrices, Bull. Aust. Math. Soc. 6 (1971) 321-326. 
[26] W.D. Wallis On the weights of Hadamard Matrices Ars Combin. 3 (1977) 287-292.

[27] J. Williamson, Hadamard's determinant theorem and the sum of four squares, Duke Math. J. 11 (1944) 65-81. 